\title{
Singular Integral Operators with Bergman-Besov Kernels on the Ball
}

\author{
H. Turgay Kaptanoğlu@ and A. Ersin Üreyen
}

\begin{abstract}
We completely characterize in terms of the six parameters involved the boundedness of all standard weighted integral operators induced by Bergman-Besov kernels acting between different Lebesgue classes with standard weights on the unit ball of $\mathbb{C}^{N}$. The integral operators generalize the Bergman-Besov projections. To find the necessary conditions for boundedness, we employ a new versatile method that depends on precise imbedding and inclusion relations among various holomorphic function spaces. The sufficiency proofs are by Schur tests or integral inequalities.
\end{abstract}

Mathematics Subject Classification. Primary 47B34, 47G10, Secondary 32A55, 45P05, 46E15, 32A37, 32A36, 30H25, 30H20.

Keywords. Integral operator, Bergman-Besov kernel, Bergman-Besov space, Bloch-Lipschitz space, Bergman-Besov projection, Radial fractional derivative, Schur test, Forelli-Rudin estimate, Inclusion relation.

\section{Introduction}

The Bergman projection is known to be a bounded operator on $L^{p}$ of the disc for all $p>1$ ever since [17]. Weighted versions in several variables are considered with the help of the Schur test in [7] resulting in projections also for $p=1$. After many modifications, integral operators similar to Bergman projections are investigated between different Lebesgue classes on the ball in several publications, such as the more recent [18].

Generalizations to other types of spaces on various domains with differing kernels are too numerous to mention here. But a complete analysis of the integral operators arising from Bergman kernels between Lebesgue classes is rather new and is attempted in [3] on the disc and for one single kernel in [2] on the ball. Here we undertake and complete the task of extending and generalizing their work to the ball, to weighted operators, to all Bergman-Besov kernels, and to Lebesgue classes with standard weights but with different exponents. Many of our results are new even in the disc. 
We present our results after giving a minimal amount of notation. Let $\mathbb{B}$ be the unit ball in $\mathbb{C}^{N}$ with respect to the norm $|z|=\sqrt{\langle z, z\rangle}$ induced by the inner product $\langle w, z\rangle=w_{1} \bar{z}_{1}+\cdots+w_{N} \bar{z}_{N}$, which is the unit disc $\mathbb{D}$ for $N=1$. Let $H(\mathbb{B})$ and $H^{\infty}$ denote the spaces of all and bounded holomorphic functions on $\mathbb{B}$, respectively.

We let $\nu$ be the Lebesgue measure on $\mathbb{B}$ normalized so that $\nu(\mathbb{B})=1$. For $q \in \mathbb{R}$, we also define on $\mathbb{B}$ the measures

$$
d \nu_{q}(z):=\left(1-|z|^{2}\right)^{q} d \nu(z) .
$$

These measures are finite for $q>-1$ and $\sigma$-finite otherwise. For $0<p<\infty$, we denote the Lebesgue classes with respect to $\nu_{q}$ by $L_{q}^{p}$, writing also $L^{p}=L_{0}^{p}$. The Lebesgue class $L_{q}^{\infty}$ of essentially bounded functions on $\mathbb{B}$ with respect to any $\nu_{q}$ is the same (see [10, Proposition 2.3]), so we denote them all by $L^{\infty}$.

Definition 1.1. For $q \in \mathbb{R}$ and $w, z \in \mathbb{B}$, the Bergman-Besov kernels are

$$
K_{q}(w, z):= \begin{cases}\frac{1}{(1-\langle w, z\rangle)^{1+N+q}}=\sum_{k=0}^{\infty} \frac{(1+N+q)_{k}}{k !}\langle w, z\rangle^{k}, & q>-(1+N), \\ { }_{2} F_{1}(1,1 ; 1-(N+q) ;\langle w, z\rangle)=\sum_{k=0}^{\infty} \frac{k !\langle w, z\rangle^{k}}{(1-(N+q))_{k}}, & q \leq-(1+N),\end{cases}
$$

where ${ }_{2} F_{1} \in H(\mathbb{D})$ is the Gauss hypergeometric function and $(u)_{v}$ is the Pochhammer symbol. They are the reproducing kernels of Hilbert BergmanBesov spaces.

The kernels $K_{q}$ for $q>-(1+N)$ can also be written as ${ }_{2} F_{1}(1,1+N+q ; 1)$ to complete the picture. The kernels $K_{q}$ for $q<-(1+N)$ appear in the literature first in $[1$, p. 13]. Notice that

$$
K_{-(1+N)}(w, z)=\frac{1}{\langle w, z\rangle} \log \frac{1}{1-\langle w, z\rangle} .
$$

For $a, b \in \mathbb{R}$, the operators acting from $L_{q}^{p}$ to $L_{Q}^{P}$ that we investigate are

$$
T_{a b} f(w)=\int_{\mathbb{B}} K_{a}(w, z) f(z)\left(1-|z|^{2}\right)^{b} d \nu(z)
$$

and

$$
S_{a b} f(w)=\int_{\mathbb{B}}\left|K_{a}(w, z)\right| f(z)\left(1-|z|^{2}\right)^{b} d \nu(z) .
$$

Our main results are the following two theorems that describe their boundedness in terms of the 6 parameters $(a, b, p, q, P, Q)$ involved. Note that at the endpoints when $p$ or $P$ is 1 or $\infty$ in Theorem 1.2 , the inequalities of (III) take several different forms. They are described in more detail in Remark 1.4 immediately following. We use $S_{a b}$ solely because we need operators with positive kernels in Schur tests.

Theorem 1.2. Let $a, b, q, Q \in \mathbb{R}, 1 \leq p \leq P \leq \infty$, and assume $Q>-1$ when $P<\infty$. Then the conditions (I), (II), (III) are equivalent.

(I) $T_{a b}: L_{q}^{p} \rightarrow L_{Q}^{P}$ is bounded.

(II) $S_{a b}: L_{q}^{p} \rightarrow L_{Q}^{P}$ is bounded. 
(III) $\frac{1+q}{p}<1+b$ and $a \leq b+\frac{1+N+Q}{P}-\frac{1+N+q}{p}$ for $1<p \leq P<\infty$;

$\frac{1+q}{p} \leq 1+b$ and $a \leq b+\frac{1+N+Q}{P}-\frac{1+N+q}{p}$ for $1=p \leq P \leq \infty$, but at least one inequality must be strict;

$\frac{1+q}{p}<1+b$ and $a<b+\frac{1+N+Q}{P}-\frac{1+N+q}{p}$ for $1<p \leq P=\infty$.

Theorem 1.3. Let $a, b, q, Q \in \mathbb{R}, 1 \leq P<p \leq \infty$, and assume $Q>-1$. Then the conditions (I), (II), (III) are equivalent.

(I) $T_{a b}: L_{q}^{p} \rightarrow L_{Q}^{P}$ is bounded.

(II) $S_{a b}: L_{q}^{p} \rightarrow L_{Q}^{P}$ is bounded.

(III) $\frac{1+q}{p}<1+b$ and $a<b+\frac{1+Q}{P}-\frac{1+q}{p}$.

Remark 1.4. When $p$ or $P$ is 1 or $\infty$, clearly the inequalities in (III) get simplified by cancellation or by $1 / \infty=0$. Considering all possible relative values of $p$ and $P$, there are 10 distinct cases each of which requiring a somewhat different proof, 6 cases for Theorem 1.2 and 4 cases for Theorem 1.3. We list below all ten of them and the exact form of (III) for each, while (I) and (II) staying the same as above. The cases $\langle 1\rangle$ and $\langle 7\rangle$ are the generic cases for $p \leq P$ and $P<p$, respectively, and the other 8 cases are the endpoints at 1 or $\infty$.

$\langle 1\rangle 1<p \leq P<\infty$ : (III) $\frac{1+q}{p}<1+b$ and $a \leq b+\frac{1+N+Q}{P}-\frac{1+N+q}{p}$.

$\langle 2\rangle 1=p=P$ : (III) $q \leq b$ and $a \leq b+Q-q$, but not both = simultaneously.

$\langle 3\rangle 1=p<P<\infty$ : (III) $q \leq b$ and $a \leq b+\frac{1+N+Q}{P}-(1+N+q)$, but not both $=$ simultaneously.

$\langle 4\rangle 1=p<P=\infty$ : (III) $q \leq b$ and $a \leq b-(1+N+q)$, but not both $=$ simultaneously.

$\langle 5\rangle 1<p<P=\infty$ : (III) $\frac{1+q}{p}<1+b$ and $a<b-\frac{1+N+q}{p}$.

$\langle 6\rangle p=P=\infty$ : (III) $0<1+b$ and $a<b$.

$\langle 7\rangle 1<P<p<\infty$ : (III) $\frac{1+q}{p}<1+b$ and $a<b+\frac{1+Q}{P}-\frac{1+q}{p}$.

$\langle 8\rangle 1=P<p<\infty$ : (III) $\frac{1+q}{p}<1+b$ and $a<b+(1+Q)-\frac{1+q}{p}$.

$\langle 9\rangle 1=P<p=\infty$ : (III) $0<1+b$ and $a<b+(1+Q)$.

$\langle 10\rangle 1<P<p=\infty$ : (III) $0<1+b$ and $a<b+\frac{1+Q}{P}$.

In the proofs, "Necessity" refers to the implication (I) $\Rightarrow$ (III), and "Sufficiency" to the implication (III) $\Rightarrow($ II). The implication (II) $\Rightarrow$ (I) is obvious.

Remark 1.5. The condition $Q>-1$ when $P<\infty$ in Theorems 1.2 and 1.3 cannot be removed as we explain in Corollary 4.11 below. This condition arises from the fact that $T_{a b}$ generates holomorphic functions and $\left|T_{a b} f\right|^{P}$ is subharmonic for $P<\infty$. It is important to note that this condition does not put any extra constraint when $P=\infty$ since $L_{Q}^{\infty}=L^{\infty}$ for any $Q$. It is no surprise that those terms in the inequalities in (III) that contain $Q$ disappear when $P=\infty$. This phenomenon occurs in the cases $\langle 4\rangle,\langle 5\rangle,\langle 6\rangle$, in which $Q \in \mathbb{R}$. So $Q>-1$ is meaningful in the remaining 7 cases.

Remark 1.6. When $a=Q$ in the case $\langle 2\rangle$, when $a=(1+N+Q) / P-(1+N)$ in the case $\langle 3\rangle$, and when $a=-(1+N)$ in the case $\langle 4\rangle$, the two inequalities 
in (III) are the same and are $q \leq b$. In such cases, $q=b$ cannot hold as stated above and proved in Theorem 6.3 below. So for these special values of $a$, we have (III) $q<b$ in the cases $\langle 2\rangle,\langle 3\rangle,\langle 4\rangle$.

Remark 1.\%. If $a \leq-(1+N)$, then the first inequality in Theorem 1.2 (III) implies the second. Indeed, the given conditions and the first inequality imply

$$
\frac{1+N+Q}{P}-a \geq \frac{N}{P}+1+N>1+N \geq \frac{1+q}{p}-b+\frac{N}{p},
$$

in which the last inequality is strict in the cases $\langle 5\rangle,\langle 6\rangle$, whence the second inequality. Similarly, if $a \leq-1$, then the first inequality in Theorem 1.3 (III) implies the second. Indeed, the given conditions and the first inequality imply $(1+Q) / P-a>0+1>(1+q) / p-b$, whence the second inequality.

The special case with $N=1, a>-2, b=0$, and $q=Q=0$ of both Theorems 1.2 and 1.3 appear in [3, Theorems 1, 2, 3, 4]; also the more restricted case with $a=-N, b=0$, and $q=Q=0$ in [2, Theorem 2]. These two references do not consider any kernels of ours for $a \leq-(1+N)$. Those parts of only Theorem 1.2 with $a>-(1+N), q>-1$, and $P<\infty$ appear in [18, Theorems 3 and 4], and its parts with $a>-(1+N)$ and $p=P=\infty$ in [11, Theorem 7.2].

Everything else in Theorems 1.2 and 1.3 is new, even for $N=1$. Thus we present a complete picture on $\mathbb{B}$ as far as the standard weights are concerned.

In [18], the kernels considered for $q \leq-(1+N)$ are simply the binomial form of $K_{q}(w, z)$ with powers negated, but these kernels are not natural in the sense that they are not positive definite, and hence cannot be reproducing kernels of Hilbert spaces; see [6, Lemma 5.1] or [9, Corollary 6.3]. Our kernels are the reproducing kernels of the Hilbert Bergman-Besov spaces $B_{q}^{2}$ and thus are positive definite. In particular, [18] does not consider a logarithmic kernel. But see [18] also for further references to earlier results.

In $[4$, Theorem 1.2], the authors prove a result similar to the sufficiency of Theorem 1.2 for $T_{a b}$ with parameters corresponding to $a=0, b \geq 0$, and $q=Q=0$ on the more general smoothly bounded, strongly pseudoconvex domains. When they further restrict to $N=1$, to $\mathbb{D}$, and to $1<p<\infty$ keeping $a=0, b \geq 0$, and $q=Q=0$, they also obtain the necessity result of Theorem 1.2. They further discuss that the second inequality in Theorem 1.2 (III) may not depend on $N$, but it turns out here that it does. We do not attempt to survey the large literature on more general domains or on more general weights. We do not also try to estimate the norms of the main operators.

However, we do consider a variation of Theorems 1.2 and 1.3 that removes the annoying condition $Q>-1$ when $P<\infty$. We achieve this by mapping $T_{a b}$ into the Bergman-Besov spaces $B_{Q}^{P}$ (see Sect. 3 and in particular Definition 3.2) instead of the Lebesgue classes. In conjuction with the claim $T_{a b} f \in H(\mathbb{B})$ of Corollary 4.11, this variation seems quite natural.

Theorem 1.8. Supppose $a, b, q, Q \in \mathbb{R}, 1 \leq p \leq \infty$, and $1 \leq P<\infty$. Then $T_{a b}: L_{q}^{p} \rightarrow B_{Q}^{P}$ is bounded if and only if the two inequalities in (III) hold in the 7 cases with $P<\infty$, that is, excluding the cases $\langle 4\rangle,\langle 5\rangle,\langle 6\rangle$. 
The very particular case of Theorem 1.8 in which $a=-N, b=0, q=0$, $Q=-N$, and $P=2$ is in [2, Theorem 1]. The following can be considered as the $P=\infty$ version of Theorem 1.8 , but it hardly says anything new in view of Corollary 4.11.

Theorem 1.9. Supppose $a, b, q \in \mathbb{R}$ and $1 \leq p \leq \infty$. Then $T_{a b}: L_{q}^{p} \rightarrow H^{\infty}$ is bounded if and only if the two inequalities in (III) hold in the 3 cases with $P=\infty$, that is, in the cases $\langle 4\rangle,\langle 5\rangle,\langle 6\rangle$.

Unlike earlier work, methods of proof we employ are uniform throughout the ten cases. The sufficiency proofs are either by Schur tests or by direct Hölder or Minkowski type inequalities which also make use of growth rate estimates of Forelli-Rudin type integrals. The necessity proofs are by an original technique that heavily depends on the precise imbedding and inclusion relations among holomorphic function spaces on $\mathbb{B}$. This technique has the potential to be used also with other kernels and spaces. By contrast, we do not use any results on Carleson measures or coefficient multipliers employed in earlier works. Our new technique is also the reason why we give all the proofs in detail, including those particular cases that are proved elsewhere by other means. It makes this paper more or less self-contained apart from some standard results and the inclusion relations.

The proofs of Theorems 1.2 and 1.3 are rather long and are presented late, necessity parts in Sect. 6 and sufficiency parts in Sect. 7. The proofs of Theorems 1.8 and 1.9 occupy Sect. 8. Before the proofs, we list the major standard results we use in Sect. 5. Earlier in Sect. 4, we place the main operators in context and develop their elementary properties. It is also here that we obtain the condition $Q>-1$. Section 3 covers the necessary background on Bergman-Besov spaces. In the next Sect. 2, we exhibit the regions of boundedness of the main operators graphically.

\section{Graphical Representation}

The repeated terms in the inequalities in (III) of Theorems 1.2 and 1.3 suggest that the 6 parameters in them can be combined in interesting ways and the region of boundedness of $T_{a b}: L_{q}^{p} \rightarrow L_{Q}^{P}$ can be described geometrically with fewer variables. In this direction, we let

$$
x=\frac{1+q}{p}-b \quad \text { and } \quad y=\frac{1+Q}{P}-a .
$$

In [3], such a region of boundedness is graphed in the $1 / p-1 / P$-plane and it is almost the same as our $x y$-plane in the absence of $b, q, Q$ and with $N=1$. With 4 extra parameters and more freedom for $a$, something similar is still possible.

There are natural bounds for $x, y$ imposed by the bounds $1 \leq p, P \leq \infty$. Since also $Q>-1$, the region of boundedness of $T_{a b}$ lies in the rectangle $R$ determined by $-a \leq y \leq 1+Q-a$ and one of $-b \leq x \leq 1+q-b$ and $1+q-b \leq x \leq-b$ depending on the sign of $1+q$. Otherwise $R$ is free to 


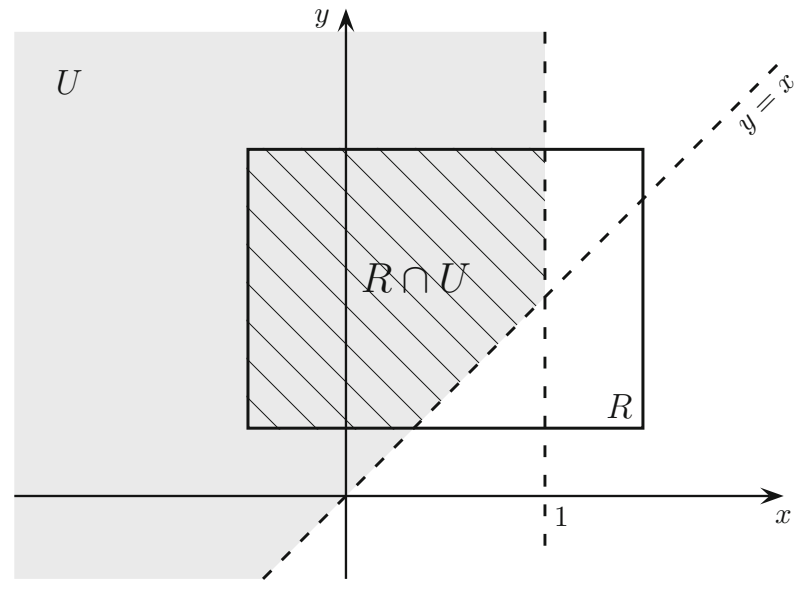

FiguRE 1. A typical region $R \cap U$

move in the $x y$-plane. Note that $R$ degenerates to a vertical line segment at $x=-b$ when $q=-1$.

In Theorem 1.3, the inequalities of (III) can now be written in the form $x<1$ and $x<y$. Each inequality determines a half plane whose intersection we call $U$. For $P<p$, the operator $T_{a b}: L_{q}^{p} \rightarrow L_{Q}^{P}$ is bounded precisely when $(x, y) \in R \cap U$. The intersection $R \cap U$ can be triangular, quadrilateral, or pentagonal, or as simple as a vertical line segment. Part of Remark 1.7 is clearer now since once $y>1$, if $R$ is to the left of $x=1$, then it is also above $y=x$. A typical $R \cap U$ is illustrated in Fig. 1 .

In Theorem 1.2, the inequalities of (III), say in the case $\langle 1\rangle$, can now be written in the form $x<1$ and $x+d \leq y$, where $d=N\left(\frac{1}{p}-\frac{1}{P}\right) \geq 0$. Each inequality again determines a half plane whose intersection we call $V$. For $p \leq P$, the operator $T_{a b}: L_{q}^{p} \rightarrow L_{Q}^{P}$ is bounded precisely when $(x, y) \in R \cap V$. The shape of $R \cap V$ is like that of $R \cap U$, but now $R \cap V$ can also be a single point when the upper left corner of $R$ lies on the line $y=x+d$. This happens only in the case $\langle 2\rangle$ with $1=p=P$ and hence $d=0$, and the minimum value of $x$ equaling the maximum value of $y$ in $R$. So with $1+q<0$, we have $1+q-b=1+Q-a$, which is actually the equality in the second inequality of (III). Then the first inequality in (III) must be strict and be $q<b$. Of course $Q>-1$. So a single point in the $x y$-plane need not correspond to a single set of values for the six parameters. This phenomenon does not occur for $P<p$ since both inequalities in (III) are then strict. The other part of Remark 1.7 is clearer now since once $y>1+N \geq 1+d$, if $R$ is to the left of $x=1$, then it is also above $y=x+d$. A typical $R \cap V$ is illustrated in Fig. 2 .

The form of the variables $x, y$ brings to mind whether or not our results on weighted spaces can be obtained from those on unweighted spaces with $q=Q=0$. It turns out that they can and we explain how in Remark 8.1. However, our proofs are not simplified significantly with $q=Q=0$. The classification in Remark 1.4 is according to $p, P$ and there seems to be no 


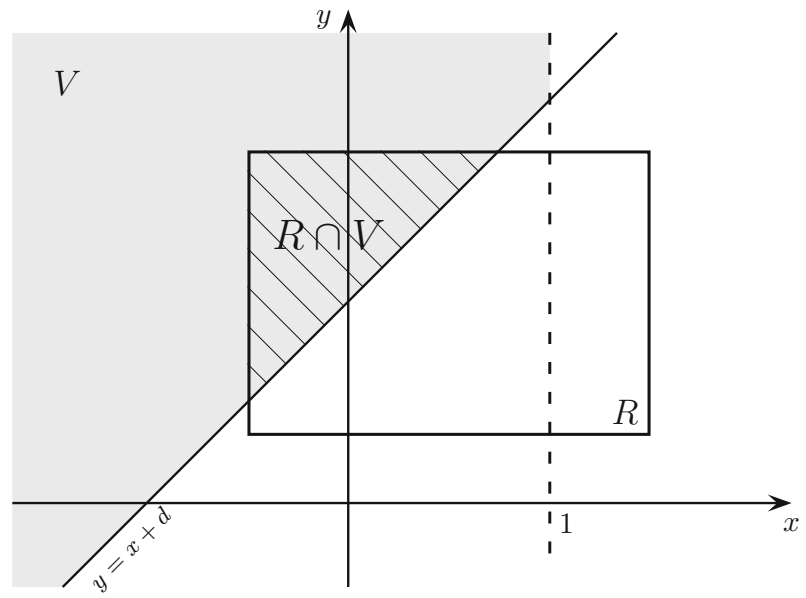

Figure 2. A typical region $R \cap V$

simple way of reducing it to fewer cases, because the inequalities in (III) can change between $<$ and $\leq$ without any apparent reason with $p, P$ and the norms on the spaces are different when $p$ or $P$ is $\infty$. Proving everything in full generality in one pass is a good idea.

\section{Preliminaries on Spaces}

We let $1 \leq p, p^{\prime} \leq \infty$ be conjugate exponents, that is, $1 / p+1 / p^{\prime}=1$, or equivalently, $p^{\prime}=p /(p-1)$. In multi-index notation, $\gamma=\left(\gamma_{1}, \ldots, \gamma_{N}\right) \in \mathbb{N}^{N}$ is an $N$-tuple of nonnegative integers, $|\gamma|=\gamma_{1}+\cdots+\gamma_{N}, \gamma !=\gamma_{1} ! \cdots \gamma_{N}$ !, $0^{0}=1$, and $z^{\gamma}=z_{1}^{\gamma_{1}} \cdots z_{N}^{\gamma_{N}}$.

Let $\mathbb{S}$ be the unit sphere in $\mathbb{C}^{N}$, which is the unit circle $\mathbb{T}$ when $N=1$. We let $\sigma$ be the Lebesgue measure on $\mathbb{S}$ normalized so that $\sigma(\mathbb{S})=1$. The polar coordinates formula that relates $\sigma$ and $\nu$ as given in $[16, \S 1.4 .3]$ is

$$
\int_{\mathbb{B}} f(z) d \nu(z)=2 N \int_{0}^{1} r^{2 N-1} \int_{\mathbb{S}} f(r \zeta) d \sigma(\zeta) d r,
$$

in which $z=r \zeta$, and we also use $w=\rho \eta$ with $\zeta, \eta \in \mathbb{S}$ and $r, \rho \geq 0$.

For $\alpha \in \mathbb{R}$, we also define the weighted classes

$$
\mathcal{L}_{\alpha}^{\infty}:=\left\{\varphi \text { measurable on } \mathbb{B}:\left(1-|z|^{2}\right)^{\alpha} \varphi(z) \in L^{\infty}\right\}
$$

so that $\mathcal{L}_{0}^{\infty}=L^{\infty}$, which are normed by

$$
\|\varphi\|_{\mathcal{L}_{\alpha}^{\infty}}:=\operatorname{essip}_{z \in \mathbb{B}}\left(1-|z|^{2}\right)^{\alpha}|\varphi(z)| .
$$

The norm on $L^{\infty}$ carries over to $H^{\infty}$ with sup.

We show an integral inner product on a space $X$ of functions by $[\cdot, \cdot]_{X}$. 
Let's explain the notation used in Definition 1.1. The Pochhammer symbol $(u)_{v}$ is defined by

$$
(u)_{v}:=\frac{\Gamma(u+v)}{\Gamma(u)}
$$

when $u$ and $u+v$ are off the pole set $-\mathbb{N}$ of the gamma function $\Gamma$. In particular, $(u)_{0}=1$ and $(u)_{k}=u(u+1) \cdots(u+k-1)$ for a positive integer $k$. The Stirling formula yields

$$
\frac{\Gamma(t+u)}{\Gamma(t+v)} \sim t^{u-v}, \quad \frac{(u)_{t}}{(v)_{t}} \sim t^{u-v}, \quad \frac{(t)_{u}}{(t)_{v}} \sim t^{u-v} \quad(\operatorname{Re} t \rightarrow \infty),
$$

where $x \sim y$ means both $x=\mathcal{O}(y)$ and $y=\mathcal{O}(x)$ for all $x, y$ in question. If only $x=\mathcal{O}(y)$, we write $x \lesssim y$. A constant $C$ may attain a different value at each occurrence. The Gauss hypergeometric function ${ }_{2} F_{1} \in H(\mathbb{D})$ is defined by

$$
{ }_{2} F_{1}(u, v ; t ; z)=\sum_{k=0}^{\infty} \frac{(u)_{k}(v)_{k}}{(t)_{k}(1)_{k}} z^{k} .
$$

A large part of this work depends on the interactions between the Lebesgue classes and the Bergman-Besov spaces. Given $q \in \mathbb{R}$ and $0<p<$ $\infty$, let $m$ be a nonnegative integer such that $q+p m>-1$. In more common notation, the Bergman-Besov space $B_{q}^{p}$ consists of all $f \in H(\mathbb{B})$ for which

$$
\left(1-|z|^{2}\right)^{m} \frac{\partial^{m} f}{\partial z_{1}^{\gamma_{1}} \cdots \partial z_{N}^{\gamma_{N}}} \in L_{q}^{p}
$$

for every multi-index $\gamma=\left(\gamma_{1}, \ldots, \gamma_{N}\right)$ with $\gamma_{1}+\cdots+\gamma_{N}=m$.

Likewise, given $\alpha \in \mathbb{R}$, let $m$ be a nonnegative integer so that $\alpha+m>0$. The Bloch-Lipschitz space $\mathcal{B}_{\alpha}^{\infty}$ consists of all $f \in H(\mathbb{B})$ for which

$$
\left(1-|z|^{2}\right)^{m} \frac{\partial^{m} f}{\partial z_{1}^{\gamma_{1}} \cdots \partial z_{N}^{\gamma_{N}}} \in \mathcal{L}_{\alpha}^{\infty}
$$

for every multi-index $\gamma=\left(\gamma_{1}, \ldots, \gamma_{N}\right)$ with $\gamma_{1}+\cdots+\gamma_{N}=m$.

However, partial derivatives are more difficult to use in the context of this paper and we follow an equivalent path. So we now introduce the radial fractional derivatives that not only allow us to define the holomorphic variants of the $L_{q}^{p}$ spaces more easily, but also form some of the most useful operators in this paper.

First let the coefficient of $\langle w, z\rangle^{k}$ in the series expansion of $K_{q}(w, z)$ in Definition 1.1 be $c_{k}(q)$. So

$$
K_{q}(w, z)=\sum_{k=0}^{\infty} c_{k}(q)\langle w, z\rangle^{k} \quad(q \in \mathbb{R}),
$$

where evidently the series converges absolutely and uniformly when one of the variables $w, z$ lies in a compact subset of $\mathbb{B}$. Note that $c_{0}(q)=1, c_{k}(q)>0$ for any $k$, and by (1),

$$
c_{k}(q) \sim k^{N+q} \quad(k \rightarrow \infty),
$$


for every $q$. This explains the choice of the parameters of the hypergeometric function in $K_{q}$.

Definition 3.1. Let $f \in H(\mathbb{B})$ be given on $\mathbb{B}$ by its convergent homogeneous expansion $f=\sum_{k=0}^{\infty} f_{k}$ in which $f_{k}$ is a homogeneous polynomial in $z_{1}, \ldots, z_{N}$ of degree $k$. For any $s, t \in \mathbb{R}$, we define the radial fractional differential operator $D_{s}^{t}$ on $H(\mathbb{B})$ by

$$
D_{s}^{t} f:=\sum_{k=0}^{\infty} d_{k}(s, t) f_{k}:=\sum_{k=0}^{\infty} \frac{c_{k}(s+t)}{c_{k}(s)} f_{k} .
$$

Note that $d_{0}(s, t)=1$ so that $D_{s}^{t}(1)=1, d_{k}(s, t)>0$ for any $k$, and

$$
d_{k}(s, t) \sim k^{t} \quad(k \rightarrow \infty),
$$

for any $s, t$ by $(3)$. So $D_{s}^{t}$ is a continuous operator on $H(\mathbb{B})$ and is of order $t$. In particular, $D_{s}^{t} z^{\gamma}=d_{|\gamma|}(s, t) z^{\gamma}$ for any multi-index $\gamma$. More importantly,

$$
D_{s}^{0}=I, \quad D_{s+t}^{u} D_{s}^{t}=D_{s}^{t+u}, \quad \text { and } \quad\left(D_{s}^{t}\right)^{-1}=D_{s+t}^{-t}
$$

for any $s, t, u$, where the inverse is two-sided. Thus any $D_{s}^{t}$ maps $H(\mathbb{B})$ onto itself.

Consider now the linear transformation $I_{s}^{t}$ defined for $f \in H(\mathbb{B})$ by

$$
I_{s}^{t} f(z):=\left(1-|z|^{2}\right)^{t} D_{s}^{t} f(z) .
$$

Definition 3.2. For $q \in \mathbb{R}$ and $0<p<\infty$, we define the Bergman-Besov space $B_{q}^{p}$ to consist of all $f \in H(\mathbb{B})$ for which $I_{s}^{t} f$ belongs to $L_{q}^{p}$ for some $s, t$ satisfying

$$
q+p t>-1 .
$$

It is well-known that under (6), Definition 3.2 is independent of $s, t$ and the norms $\|f\|_{B_{q}^{p}}:=\left\|I_{s}^{t} f\right\|_{L_{q}^{p}}$ are all equivalent. Explicitly,

$$
\|f\|_{B_{q}^{p}}^{p}=\int_{\mathbb{B}}\left|D_{s}^{t} f(z)\right|^{p}\left(1-|z|^{2}\right)^{q+p t} d \nu(z) \quad(q+p t>-1) .
$$

When $q>-1$, we can take $t=0$ in (6) and obtain the weighted Bergman spaces $A_{q}^{p}=B_{q}^{p}$. We also wite $A^{p}=A_{0}^{p}$. For $0<p<1$, what we call norms are actually quasinorms.

Definition 3.3. For $\alpha \in \mathbb{R}$, we define the Bloch-Lipschitz space $\mathcal{B}_{\alpha}^{\infty}$ to consist of all $f \in H(\mathbb{B})$ for which $I_{s}^{t} f$ belongs to $\mathcal{L}_{\alpha}^{\infty}$ for some $s, t$ satisfying

$$
\alpha+t>0 \text {. }
$$

It is well-known that under (8), Definition 3.3 is independent of $s, t$ and the norms $\|f\|_{\mathcal{B}_{\alpha}^{\infty}}:=\left\|I_{s}^{t} f\right\|_{\mathcal{L}_{\alpha}^{\infty}}$ are all equivalent. Explicitly,

$$
\|f\|_{\mathcal{B}_{\alpha}^{\infty}}=\sup _{z \in \mathbb{B}}\left|D_{s}^{t} f(z)\right|\left(1-|z|^{2}\right)^{\alpha+t} \quad(\alpha+t>0) .
$$

If $\alpha>0$, we can take $t=0$ in (8) and obtain the weighted Bloch spaces. When $\alpha<0$, then the corresponding spaces are the holomorphic Lipschitz spaces $\Lambda_{-\alpha}=\mathcal{B}_{\alpha}^{\infty}$. The usual Bloch space $\mathcal{B}_{0}^{\infty}=\mathcal{B}^{\infty}$ corresponds to $\alpha=0$. 
Admittedly this notation is a bit unusual, but there is no mention of little Bloch spaces in this paper.

It is also well-known that a Bergman-Besov space defined using partial derivatives or radial fractional differential operators contain the same functions. The same is true also for a Bloch-Lipschitz space.

Remark 3.4. Definitions 3.2 and 3.3 imply that $I_{s}^{t}$ imbeds $B_{q}^{p}$ isometrically into $L_{q}^{p}$ if and only if (6) holds, and $I_{s}^{t}$ imbeds $\mathcal{B}_{\alpha}^{\infty}$ isometrically into $\mathcal{L}_{\alpha}^{\infty}$ if and only if (8) holds.

Much more information about the spaces $B_{q}^{p}$ and $\mathcal{B}_{\alpha}^{\infty}$ and their interconnections can be found in [12].

\section{Properties of Kernels and Operators}

It is well-known that every $B_{q}^{2}$ space is a reproducing kernel Hilbert space and its reproducing kernel is $K_{q}$. Thus $K_{q}(w, z)$ is positive definite for $w, z \in \mathbb{B}$. Further, $K_{q}(w, z)$ is holomorphic in $w \in \mathbb{B}$ and satisfy $\overline{K_{q}(z, w)}=K_{q}(w, z)$. In particular, for $q>-1$, the $B_{q}^{2}$ are weighted Bergman spaces $A_{q}^{2}, B_{-1}^{2}$ is the Hardy space $H^{2}, B_{-N}^{2}$ is the Drury-Arveson space, and $B_{-(1+N)}^{2}$ is the Dirichlet space. Moreover, the norm on $B_{q}^{2}$ obtained from its reproducing kernel $K_{q}$ is equivalent to its integral norms given in (7).

For ease of writing, put $\omega=\langle w, z\rangle \in \mathbb{D}$ and let $k_{q}(\omega)=K_{q}(w, z)$, which is holomorphic in $\omega \in \mathbb{D}$.

Lemma 4.1. For $q<-(1+N)$, each $\left|K_{q}(w, z)\right|$ is bounded above as $w, z$ vary in $\mathbb{B}$. More importantly, each $\left|K_{q}(w, z)\right|$ with $q \in \mathbb{R}$ is bounded below by a positive constant as $w, z$ vary in $\mathbb{B}$. Therefore no $K_{q}$ has a zero in $\mathbb{B} \times \mathbb{B}$.

Proof. When $q<-(1+N)$, the growth rate $(3)$ of the coefficient $c_{k}$ of $\omega^{k}$ shows that the power series of $k_{q}(\omega)$ converges uniformly for $\omega \in \overline{\mathbb{D}}$. This shows boundedness. Next we consult [15, Equation 15.13.1] and see that $k_{q}$ is not 0 on a set containing $\overline{\mathbb{D}} \backslash\{1\}$. The reason for this is that the first parameter 1 of the hypergeometric function defining $k_{q}$ is positive. But also $k_{q}(1) \neq 0$ clearly. Thus $\left|k_{q}\right|$ is bounded below on $\overline{\mathbb{D}}$.

If $q=-(1+N)$, then $k_{-(1+N)}(\omega)=\omega^{-1} \log (1-\omega)^{-1}$. On $\overline{\mathbb{D}} \backslash\{1\}$, $k_{-(1+N)}$ is not zero and $\left|k_{q}(\omega)\right|$ blows up as $\omega \rightarrow 1$ within $\overline{\mathbb{D}}$. So $\left|k_{q}\right|$ is bounded below on $\overline{\mathbb{D}}$.

The claim about $\left|K_{q}\right|$ for $q>-(1+N)$ is obvious and the lower bound can be taken as $2^{-(1+N+q)}$.

One of the best things about the radial differential operators $D_{s}^{t}$ is that they allow us to pass easily from one kernel to another and from one space to another in the same family. First, it is immediate that

$$
D_{q}^{t} K_{q}(w, z)=K_{q+t}(w, z) \quad(q, t \in \mathbb{R}),
$$

where differentiation is performed on the holomorphic variable $w$. But the more versatile result is the following, which is a combination of [11, Proposition 3.2 and Corollary 8.5]. 
Theorem 4.2. Let $q, \alpha, s, t \in \mathbb{R}$ and $0<p<\infty$ be arbitrary. Then the maps $D_{s}^{t}: B_{q}^{p} \rightarrow B_{q+p t}^{p}$ and $D_{s}^{t}: \mathcal{B}_{\alpha}^{\infty} \rightarrow \mathcal{B}_{\alpha+t}^{\infty}$ are isomorphisms, and isometries when the parameters of the norms of the spaces are chosen appropriately.

Definition 4.3. For $b \in \mathbb{R}$, the Bergman-Besov projections are the operators $T_{b b}$ acting on a suitable Lebesgue class with range in a holomorphic function space, both on $\mathbb{B}$.

The following general result describes the boundedness of BergmanBesov projections on all two-parameter Bergman-Besov spaces and the usual Bloch space, and is [10, Theorem 1.2]. It is also an indicator of the importance of the operators $I_{s}^{t}$ which we use repeatedly in this paper.

Theorem 4.4. For $q \in \mathbb{R}$ and $1 \leq p<\infty$, the $T_{b b}: L_{q}^{p} \rightarrow B_{q}^{p}$ is bounded if and only if

$$
\frac{1+q}{p}<1+b
$$

And $T_{b b}: L^{\infty} \rightarrow \mathcal{B}^{\infty}$ is bounded if and only if

$$
0<1+b \text {. }
$$

Moreover, given b satisfying (10), if $t$ satisfies (6), then $T_{b b} I_{b}^{t}=C f$ for $f \in B_{q}^{p}$. And given $b$ satisfying (11), if $t$ satisfies (8) with $\alpha=0$, then $T_{b b} I_{b}^{t} f=C f$ for $f \in \mathcal{B}^{\infty}$. Here $C$ is a constant that depends on $N, b, t$, but not on $f$.

The next lemma is adapted from [5, Lemma 3.2]. To see what it means, first check that $K_{q}(0, z)=1$ for all $z \in \mathbb{B}$.

Lemma 4.5. For each $q \in \mathbb{R}$, there is a $\rho_{0}<1$ such that for $|w| \leq \rho_{0}$ and all $z \in \mathbb{B}$, we have $\operatorname{Re} K_{q}(w, z) \geq 1 / 2$.

Proof. By $(3),\left|K_{q}(w, z)\right| \leq 1+C \sum_{k=1}^{\infty} k^{N+q}|\langle w, z\rangle|^{k}$ for some constant $C$ and

$$
C \sum_{k=1}^{\infty} k^{N+q}|\langle w, z\rangle|^{k} \leq C \sum_{k=1}^{\infty} k^{N+q}|w|^{k}|z|^{k} \leq C|w| \sum_{k=1}^{\infty} k^{N+q}|w|^{k-1}
$$

for all $z, w \in \mathbb{B}$. The last series converges, say, for $|w|=1 / 2$; call its sum $W$ and set $\rho_{0}=\min \{1 / 2,1 / 2 C W\}$. If $|w| \leq \rho_{0}$, then

$$
\left|C \sum_{k=1}^{\infty} k^{N+q}\langle w, z\rangle^{k}\right| \leq C W|w| \leq \frac{1}{2} \quad(z \in \mathbb{B}) .
$$

That is, $\left|K_{q}(w, z)-1\right| \leq 1 / 2$ for $|w| \leq \rho_{0}$ and all $z \in \mathbb{B}$. This implies the desired result.

We turn to the operators $T_{a b}$ and formulate their behavior in many important situations. But first we insert some obvious inequalities we use many times in the proofs. If $c<d, u>0$, and $v \in \mathbb{R}$, then for $0 \leq r<1$,

$$
\left(1-r^{2}\right)^{d} \leq\left(1-r^{2}\right)^{c} \quad \text { and } \quad\left(1-r^{2}\right)^{u}\left(\frac{1}{r^{2}} \log \frac{1}{1-r^{2}}\right)^{-v} \lesssim 1
$$

The second leads to an estimate we need several times. 
Lemma 4.6. For $u, v \in \mathbb{R}$,

$$
\int_{0}^{1}\left(1-r^{2}\right)^{u}\left(\frac{1}{r^{2}} \log \frac{1}{1-r^{2}}\right)^{-v} d r<\infty
$$

if $u>-1$ or if $u=-1$ and $v>1$, and the integral diverges otherwise.

Proof. The only singularity of the integrand is at $r=1$. For $u \neq-1$, polynomial growth dominates a logarithmic one. For $u=-1$, we reduce the integral into one studied in calculus after changes of variables.

We call

$$
f_{u v}(z)=\left(1-|z|^{2}\right)^{u}\left(\frac{1}{|z|^{2}} \log \frac{1}{1-|z|^{2}}\right)^{-v}
$$

test functions, because we derive half the necessary conditions of Theorems 1.2 and 1.3 from the action of $T_{a b}$ on them. Here $u, v \in \mathbb{R}$ and $f_{00}=1$. When we apply Lemma 4.6 to the $f_{u v}$, we obtain the next result.

Lemma 4.7. For $1 \leq p<\infty$, we have $f_{u v} \in L_{q}^{p}$ if and only if $q+p u>-1$, or $q+p u=-1$ and $p v>1$. For $p=\infty$, we have $f_{u v} \in L^{\infty}$ if and only if $u>0$, or $u=0$ and $v \geq 0$.

Lemma 4.8. If $b+u>-1$ or if $b+u=-1$ and $v>1$, then $T_{a b} f_{u v}$ is a finite positive constant. Otherwise, $\left|T_{a b} f_{u v}(w)\right|=\infty$ for $|w| \leq \rho_{0}$, where $\rho_{0}$ is as in Lemma 4.5 .

Proof. If $b+u>-1$, or $b+u=-1$ and $v>1$, then by polar coordinates and the mean value property,

$$
\begin{aligned}
T_{a b} f_{u v}(w) & =\int_{\mathbb{B}} K_{a}(w, z)\left(1-|z|^{2}\right)^{b+u}\left(\frac{1}{|z|^{2}} \log \frac{1}{1-|z|^{2}}\right)^{-v} d \nu(z) \\
& =C \int_{0}^{1} r^{2 N-1}\left(1-r^{2}\right)^{b+u}\left(\frac{1}{r^{2}} \log \frac{1}{1-r^{2}}\right)^{-v} \frac{\int_{\mathbb{S}} K_{a}(r \zeta, w) d \sigma(\zeta)}{\int^{-v}} \overline{K_{a}(0, w)} d r \\
& =C \int_{0}^{1} r^{2 N-1}\left(1-r^{2}\right)^{b+u}\left(\frac{1}{r^{2}} \log \frac{1}{1-r^{2}}\right)^{-v} d r .
\end{aligned}
$$

The last integral is finite by Lemma 4.6, and then evidently $T_{a b} f_{u v}$ is a constant.

For other values of the parameters,

$$
\begin{aligned}
\left|T_{a b} f_{u v}(w)\right| \geq \operatorname{Re} T_{a b} f_{u v}(w) & \geq \frac{1}{2} \int_{\mathbb{B}}\left(1-|z|^{2}\right)^{b+u}\left(\frac{1}{|z|^{2}} \log \frac{1}{1-|z|^{2}}\right)^{-v} d \nu(z) \\
& =\infty
\end{aligned}
$$

for $|w| \leq \rho_{0}$ by Lemma 4.5 and Lemma 4.6.

The adjoint of $T_{a b}$ is readily computed. 
Proposition 4.9. The formal adjoint $T_{a b}^{*}: L_{Q}^{P^{\prime}} \rightarrow L_{q}^{p^{\prime}}$ of $T_{a b}: L_{q}^{p} \rightarrow L_{Q}^{P}$ for $1 \leq p, P<\infty$ is $T_{a b}^{*}=M_{b-q} T_{a Q}$, where $M_{b-q}$ denotes the operator of multiplication by $\left(1-|z|^{2}\right)^{b-q}$.

Proof. Let $f \in L_{q}^{p}$ and $g \in L_{Q}^{P^{\prime}}$. Then

$$
\begin{aligned}
{\left[T_{a b} f, g\right]_{L_{Q}^{2}}=} & \int_{\mathbb{B}} \int_{\mathbb{B}} K_{a}(w, z) f(z)\left(1-|z|^{2}\right)^{b} d \nu(z) \overline{g(w)}\left(1-|w|^{2}\right)^{Q} d \nu(w) \\
= & \int_{\mathbb{B}} f(z) \overline{\left(1-|z|^{2}\right)^{b-q} \int_{\mathbb{B}} K_{a}(z, w) g(w)\left(1-|w|^{2}\right)^{Q} d \nu(w)} \\
& \cdot\left(1-|z|^{2}\right)^{q} d \nu(z) \\
= & \int_{\mathbb{B}} f \overline{T_{a b}^{*}(g)} d \nu_{q}=\left[f, T_{a b}^{*} g\right]_{L_{q}^{2} .}
\end{aligned}
$$

Thus

$$
T_{a b}^{*} g(z)=\left(1-|z|^{2}\right)^{b-q} \int_{\mathbb{B}} K_{a}(z, w) g(w)\left(1-|w|^{2}\right)^{Q} d \nu(w) .
$$

The following simple but very helpful result is well known, but we include its proof for completeness.

Lemma 4.10. If $0<P<\infty, Q \leq-1, g \in H(\mathbb{B})$, and $g \neq \equiv$, then

$$
J:=\int_{\mathbb{B}}|g(w)|^{P}\left(1-|w|^{2}\right)^{Q} d \nu(w)=\infty .
$$

Proof. We have

$$
\begin{aligned}
J & \geq 2 N \int_{1 / 2}^{1} \rho^{2 N-1}\left(1-\rho^{2}\right)^{Q} \int_{\mathbb{S}}|g(\rho \eta)|^{P} d \sigma(\eta) d \rho \\
& \gtrsim \int_{1 / 2}^{1} \frac{1}{2^{2 N-1}}\left(1-\rho^{2}\right)^{Q} \int_{\mathbb{S}}\left|g\left(\frac{\eta}{2}\right)\right|^{P} d \sigma(\eta) d \rho \gtrsim \int_{1 / 2}^{1}\left(1-\rho^{2}\right)^{Q} d \rho=\infty
\end{aligned}
$$

by polar coordinates and the subharmonicity of $|g|^{P}$.

Corollary 4.11. If $T_{a b}: L_{q}^{p} \rightarrow L_{Q}^{P}$ is bounded and $f \in L_{q}^{p}$, then $g=T_{a b} f$ lies in $H(\mathbb{B})$. If also $P<\infty$, then $Q>-1$. Thus $T_{a b}: L_{q}^{p} \rightarrow A_{Q}^{P}$ when it is bounded with $P<\infty$. Consequently, if $P<\infty$ and $Q \leq-1$, then $T_{a b}: L_{q}^{p} \rightarrow L_{Q}^{P}$ is not bounded, and hence $S_{a b}: L_{q}^{p} \rightarrow L_{Q}^{P}$ is not bounded. On the other hand, if $T_{a b}: L_{q}^{p} \rightarrow L^{\infty}$ is bounded and $f \in L_{q}^{p}$, then $T_{a b} f \in H^{\infty}$ naturally.

Proof. That $g$ is holomorphic follows, for example, by differentiation under the integral sign, from the fact that $K_{q}(w, z)$ is holomorphic in $w$. That $Q>-1$ follows from Lemma 4.10 .

Let's stress again that Corollary 4.11 does not place any restriction on $Q$ when $P=\infty$, simply because the space $L_{Q}^{P}$ and the inequalities in (III) are independent of $Q$ when $P=\infty$. 
Lemma 4.12. If $S_{c b}: L_{q}^{p} \rightarrow L_{Q}^{P}$ is bounded and $a<c$, then $S_{a b}: L_{q}^{p} \rightarrow L_{Q}^{P}$ is also bounded.

Proof. This is because $\left|K_{a}\right| \lesssim\left|K_{c}\right|$, which we now prove. If $a>-(1+N)$, as done in $[18$, p. 525], we write

$$
\frac{1}{|1-\langle w, z\rangle|^{1+N+a}}=\frac{|1-\langle w, z\rangle|^{c-a}}{|1-\langle w, z\rangle|^{1+N+c}} \leq \frac{2^{c-a}}{|1-\langle w, z\rangle|^{1+N+c}} .
$$

Let now $a=-(1+N)$. On that part of $\mathbb{D}$ away from $1,\left|k_{-(1+N)}\right|$ is bounded above and $\left|k_{c}\right|$ is bounded below by Lemma 4.1 . On that part of $\mathbb{D}$ near 1 , $\left|k_{-(1+N)}\right|$ is still dominated by $\left|k_{c}\right|$, because

$$
\lim _{\mathbb{D} \ni \omega \rightarrow 1} \frac{\omega^{-1} \log (1-\omega)^{-1}}{(1-\omega)^{-(1+N+c)}}=0 .
$$

If $a<-(1+N)$, then $\left|K_{c}\right|$ dominates $\left|K_{a}\right|$ by Lemma 4.1. Thus in all cases $\left|K_{a}(w, z)\right| \lesssim\left|K_{c}(w, z)\right|$ for all $w, z \in \mathbb{B}$ if $a<c$.

\section{Main Tools}

Let $(X, \mathcal{A}, \lambda)$ and $(Y, \mathcal{B}, \mu)$ be two measure spaces, $G(x, y)$ a nonnegative function on $X \times Y$ measurable with respect to $\mathcal{A} \times \mathcal{B}$, and let $Z$ be given by

$$
Z f(y)=\int_{X} G(x, y) f(x) d \lambda(x)
$$

The following two Schur tests are of crucial importance. The first is [13, Theorem 2.1], and is rediscovered in [18, Theorem 2].

Theorem 5.1. Let $1<p \leq P<\infty$, and suppose that there are $c, d \in \mathbb{R}$ with $c+d=1$ and strictly positive functions $\phi$ on $X$ and $\psi$ on $Y$ such that

$$
\begin{array}{ll}
\int_{X} G(x, y)^{c p^{\prime}} \phi(x)^{p^{\prime}} d \lambda(x) \lesssim \psi(y)^{p^{\prime}} & \text { a.e. }[\mu], \\
\int_{Y} G(x, y)^{d P} \psi(y)^{P} d \mu(y) \lesssim \phi(x)^{P} & \text { a.e. }[\lambda] .
\end{array}
$$

Then $Z: L^{p}(\lambda) \rightarrow L^{P}(\mu)$ is bounded.

The second is [8, Theorem 1.I].

Theorem 5.2. Let $1<P<p<\infty$, and suppose that there are strictly positive functions $\phi$ on $X$ and $\psi$ on $Y$ such that

$$
\begin{aligned}
\int_{X} G(x, y) \phi(x)^{p^{\prime}} d \lambda(x) & \lesssim \psi(y)^{P^{\prime}} \quad \text { a.e. }[\mu], \\
\int_{Y} G(x, y) \psi(y)^{P} d \mu(y) & \lesssim \phi(x)^{p} \quad \text { a.e. }[\lambda], \\
\iint_{X \times Y} G(x, y) \phi(x)^{p^{\prime}} \psi(y)^{P} d(\lambda \times \mu)(x, y) & \lesssim 1 .
\end{aligned}
$$

Then $Z: L^{p}(\lambda) \rightarrow L^{P}(\mu)$ is bounded. 
We need in one place the less known Minkowski integral inequality that in effect exchanges the order of integration; for a proof, see [14, Theorem 3.3.5] for example.

Lemma 5.3. If $1 \leq p \leq \infty$ and $f(x, y)$ is measurable with respect to $\mathcal{A} \times \mathcal{B}$, then

$$
\left(\int_{Y}\left(\int_{X}|f(x, y)| d \lambda(x)\right)^{p} d \mu(y)\right)^{1 / p} \leq \int_{X}\left(\int_{Y}|f(x, y)|^{p} d \mu(y)\right)^{1 / p} d \lambda(x),
$$

with an appropriate interpretation with the $L^{\infty}$ norm when $p=\infty$.

We cannot do without the Forelli-Rudin estimates of [16, Proposition 1.4.10].

Proposition 5.4. For $d>-1$ and $c \in \mathbb{R}$, we have

$$
\int_{\mathbb{B}} \frac{\left(1-|w|^{2}\right)^{d}}{|1-\langle z, w\rangle|^{1+N+c}} d \nu(w) \sim \begin{cases}1, & c<d, \\ |z|^{-2} \log \left(1-|z|^{2}\right)^{-1}, & c=d, \\ \left(1-|z|^{2}\right)^{-(c-d)}, & c>d .\end{cases}
$$

The following result from [10, Lemma 5.1] is extremely useful.

Lemma 5.5. If $b>-1, a \in \mathbb{R}$, and $f \in H(\mathbb{B}) \cap L_{b}^{1}$, then

$$
T_{a b} f(w)=\int_{\mathbb{B}} K_{a}(w, z) f(z)\left(1-|z|^{2}\right)^{b} d \nu(z)=\frac{N !}{(1+b)_{N}} D_{b}^{a-b} f(w) .
$$
proofs.

The important result that we prove now is indispensable in our necessity

Lemma 5.6. If $b+t>-1$, then $T_{a b} I_{b}^{t} h=C D_{b}^{a-b} h$ for $h \in B_{b}^{1}$, where $I_{b}^{t}$ is as given in (5). As consequences, $D_{a}^{b-a} T_{a b} I_{b}^{t} h=C h$ for $h \in B_{b}^{1}$ and $T_{a b} I_{b}^{t} D_{a}^{b-a}=C h$ for $h \in B_{a}^{1}$.

Proof. If $h \in B_{b}^{1}$ and $b+t>-1$, then $D_{b}^{t} h \in B_{b+t}^{1} \subset L_{b+t}^{1}$ by Theorem 4.2. Then by Lemma 5.5 and (4),

$$
\begin{aligned}
T_{a b} I_{b}^{t} h(w) & =\int_{\mathbb{B}} K_{a}(w, z) D_{b}^{t} h(z)\left(1-|z|^{2}\right)^{b+t} d \nu(z) \\
& =T_{a, b+t} D_{b}^{t} h(w)=C D_{b+t}^{a-b-t} D_{b}^{t} h(w)=C D_{b}^{a-b} h(w) .
\end{aligned}
$$

The identities on triple compositions are consequences of the identities in (4).

Here's another similar result adapted from [5, Lemma 2.3].

Lemma 5.7. If $f \in L_{b}^{1}$, then $D_{a}^{t} T_{a b} f=T_{a+t, b} f$.

Proof. By (2), if $f \in L_{b}^{1}$, then

$$
\begin{aligned}
T_{a b} f(w) & =\int_{\mathbb{B}} K_{a}(w, z) f(z) d \nu_{b}(z)=\int_{\mathbb{B}} \sum_{k=0}^{\infty} c_{k}(a)\langle w, z\rangle^{k} f(z) d \nu_{b}(z) \\
& =\sum_{k=0}^{\infty} c_{k}(a) \int_{\mathbb{B}}\langle w, z\rangle^{k} f(z) d \nu_{b}(z)=: \sum_{k=0}^{\infty} c_{k}(a) p_{k}(w),
\end{aligned}
$$


where $p_{k}$ is a holomorphic homogeneous polynomial of degree $k$. Then by Definition 3.1 and (2) again,

$$
\begin{aligned}
D_{a}^{t} T_{a b} f(w) & =\sum_{k=0}^{\infty} d_{k}(a, t) c_{k}(a) p_{k}(w)=\sum_{k=0}^{\infty} c_{k}(a+t) p_{k}(w) \\
& =\sum_{k=0}^{\infty} c_{k}(a+t) \int_{\mathbb{B}}\langle w, z\rangle^{k} f(z) d \nu_{b}(z) \\
& =\int_{\mathbb{B}} \sum_{k=0}^{\infty} c_{k}(a+t)\langle w, z\rangle^{k} f(z) d \nu_{b}(z) \\
& =\int_{\mathbb{B}} K_{a+t}(w, z) f(z) d \nu_{b}(z)=T_{a+t, b} f(w) .
\end{aligned}
$$

Above, we have used differentiation under the integral sign and (9).

The next four theorems on inclusions have been developed by various authors culminating in [12], where references to earlier work can be found. We require them in the necessity proofs. All inclusions in them are continuous, strict, and the best possible. As for notation, if $X_{v}$ is a family of spaces indexed by $v \in \mathbb{R}$, the symbol $X_{<v}$ denotes any one of the spaces $X_{u}$ with $u<v$. Let's also single out the trivial inclusions which are special cases:

$$
B_{q}^{p} \subset B_{Q}^{p} \quad(q \leq Q) \quad \text { and } \quad \mathcal{B}_{\alpha}^{\infty} \subset \mathcal{B}_{\beta}^{\infty} \quad(\alpha \leq \beta) .
$$

Theorem 5.8. Let $B_{q}^{p}$ be given.

(i) If $p \leq P$, then $B_{q}^{p} \subset B_{Q}^{P}$ if and only if $\frac{1+N+q}{p} \leq \frac{1+N+Q}{P}$.

(ii) If $P<p$, then $B_{q}^{p} \subset B_{Q}^{P}$ if and only if $\frac{1+q}{p}<\frac{1+Q}{P}$.

Theorem 5.9. (i) $H^{\infty} \subset B_{q}^{p}$ if and only if $q>-1$, or $q=-1$ and $p \geq 2$. (ii) $B_{q}^{p} \subset H^{\infty}$ if and only if $q<-(1+N)$, or $q=-(1+N)$ and $0<p \leq 1$.

Theorem 5.10. Given $B_{q}^{p}$, we have $\mathcal{B}_{<\frac{1+q}{p}}^{\infty} \subset B_{q}^{p} \subset \mathcal{B}_{\frac{1+N+q}{p}}^{\infty}$.

Theorem 5.11. $\mathcal{B}_{<0}^{\infty} \subset H^{\infty} \subset \mathcal{B}^{\infty}$.

\section{Necessity Proofs}

Now we obtain the two inequalities in (III) of Theorems 1.2 and 1.3 from the boundedness of $T_{a b}$. In this section, we do not need to assume $Q>-1$ since the boundedness of $T_{a b}$ implies $Q>-1$ when $P<\infty$ by Corollary 4.11. We first derive the first inequality in (III) of each of the 10 cases as a separate theorem. We call it the first necessary condition. The reason underlying it is understandably Theorem 4.4.

Theorem 6.1. Let $a, b, q, Q \in \mathbb{R}$ and $1 \leq p, P \leq \infty$. If $T_{a b}: L_{q}^{p} \rightarrow L_{Q}^{P}$ is bounded, then $\frac{1+q}{p} \leq 1+b$ in the cases $\langle 2\rangle,\langle 3\rangle,\langle 4\rangle$, and $\frac{1+q}{p}<1+b$ in the remaining cases. 
Proof. The ten cases can be handled in three groups depending on the value of $p$. The first group consists of the cases $\langle 1\rangle,\langle 5\rangle,\langle 7\rangle,\langle 8\rangle$ in which $1<p<\infty$. Consider $f_{u v}$ with $u=-(1+q) / p$ and $v=1$ so that $f_{u v} \in L_{q}^{p}$ by Lemma 4.7. Then $T_{a b} f_{u v} \in L_{Q}^{P}$ and Lemma 4.8 implies $b+u>-1$. With the value of $u$ chosen, this yields $(1+q) / p<1+b$.

The second group consists of the cases $\langle 2\rangle,\langle 3\rangle,\langle 4\rangle$; here $p=1$. Consider $f_{u 0}$ with $u>-(1+q)$ so that $f_{u 0} \in L_{q}^{1}$ by Lemma 4.7. Then $T_{a b} f_{u 0} \in L_{Q}^{P}$ and Lemma 4.8 implies $b+u>-1$. Writing $u=-1-q+\varepsilon$ with $\varepsilon>0$, we obtain $q<b+\varepsilon$. This is nothing but $q \leq b$.

The third group consists of the cases $\langle 6\rangle,\langle 9\rangle,\langle 10\rangle$. Now $p=\infty$ and $f_{00}=1$ is in $L^{\infty}$. Then $T_{a b} f_{00} \in L_{Q}^{P}$ and hence $0<1+b$ by Lemma 4.8 .

We next derive the second inequality in (III) of each of the 10 cases also as a separate theorem. We call it the second necessary condition. We do this by a new original method that relies on knowing which Bergman-Besov and Bloch-Lipschitz spaces lie in which others and in $H^{\infty}$. A crucial component of this method is Lemma 5.6.

Theorem 6.2. Let $a, b, q, Q \in \mathbb{R}$ and $1 \leq p, P \leq \infty$. Suppose $T_{a b}: L_{q}^{p} \rightarrow L_{Q}^{P}$ is bounded. Then $a \leq b+\frac{1+N+Q}{P}-\frac{1+N+q}{p}$ in the cases $\langle 1\rangle,\langle 2\rangle,\langle 3\rangle,\langle 4\rangle$, also $a<b+\frac{1+N+Q}{P}-\frac{1+N+q}{p}$ in the cases $\langle 5\rangle,\langle 6\rangle$, and $a<b+\frac{1+Q}{P}-\frac{1+q}{p}$ in the cases $\langle 7\rangle,\langle 8\rangle,\langle 9\rangle,\langle 10\rangle$.

Proof. The boundedness of $T_{a b}$ via Corollary 4.11 implies $Q>-1$ when $P<\infty$.

This time we form four groups of the ten cases. The first group again consists of the cases $\langle 1\rangle,\langle 7\rangle,\langle 8\rangle$, and $\langle 2\rangle,\langle 3\rangle$. Let $h \in B_{q}^{p}$. In order to be able to use Lemma 5.6, we need to make sure $h \in B_{b}^{1}$. In the cases $\langle 1\rangle,\langle 7\rangle,\langle 8\rangle$, the first necessary condition gives $(1+q) / p<1+b$, and then Theorem 5.8 (ii) shows $h \in B_{b}^{1}$. In the cases $\langle 2\rangle,\langle 3\rangle$ where $p=1$, Theorem 6.1 gives $q \leq b$, and then (13) shows $h \in B_{b}^{1}$ again. Let $t$ satisfy (6). Together with the first necessary condition, this gives $b+t>-1$. Now consider the sequence of bounded maps

$$
B_{q}^{p} \stackrel{I_{b}^{t}}{\longrightarrow} L_{q}^{p} \stackrel{T_{a b}}{\longrightarrow} L_{Q}^{P} \cap H(\mathbb{B})=A_{Q}^{P} \stackrel{D_{a}^{b-a}}{\longrightarrow} B_{Q+P(b-a)}^{P},
$$

where Theorem 4.2 is invoked and $Q>-1$ is used in writing $A_{Q}^{P}$. Lemma 5.6 yields that $B_{q}^{p}$ is imbedded in $B_{Q+P(b-a)}^{P}$ by the inclusion map. By Theorem 5.8, this implies $a<b+(1+Q) / P-(1+q) / p$ in the cases $\langle 7\rangle,\langle 8\rangle$, and it implies $a \leq b+(1+N+Q) / P-(1+N+q) / p$ in the cases $\langle 1\rangle,\langle 2\rangle,\langle 3\rangle$.

The second group consists of the cases $\langle 4\rangle,\langle 5\rangle$. Let $H \in B_{q+p(a-b)}^{p}$; then $D_{a}^{b-a} H=h \in B_{q}^{p}$ by Theorem 4.2. Exactly as in the proof of the first group of cases, $h \in B_{b}^{1}$. Let $t$ satisfy (6). Together with the first necessary condition, this gives $b+t>-1$. Now consider the sequence of bounded maps

$$
B_{q+p(a-b)}^{p} \stackrel{D_{a}^{b-a}}{\longrightarrow} B_{q}^{p} \stackrel{I_{b}^{t}}{\longrightarrow} L_{q}^{p} \stackrel{T_{a b}}{\longrightarrow} L^{\infty} \cap H(\mathbb{B})=H^{\infty} .
$$


Lemma 5.6 yields that $B_{q+p(a-b)}^{p}$ is imbedded in $H^{\infty}$ by the inclusion map. By Theorem 5.9 (ii), this implies $a<b-(1+N+q) / p$ in the case $\langle 5\rangle$, and it implies $a \leq b-(1+N+q)$ in the case $\langle 4\rangle$.

The third group consists of the cases $\langle 9\rangle,\langle 10\rangle$. Let $h \in \mathcal{B}^{\infty}$. Theorem 6.1 gives $0<1+b$, and then Theorem 5.10 yields $h \in B_{b}^{1}$. Let $t>0$, that is, satisfy (8) with $\alpha=0$. Together with the first necessary condition, this gives $b+t>-1$. Now consider the sequence of bounded maps

$$
\mathcal{B}^{\infty} \stackrel{I_{b}^{t}}{\longrightarrow} L^{\infty} \stackrel{T_{a b}}{\longrightarrow} L_{Q}^{P} \cap H(\mathbb{B})=A_{Q}^{P} \stackrel{D_{a}^{b-a}}{\longrightarrow} B_{Q+P(b-a)}^{P},
$$

where Theorem 4.2 is invoked and $Q>-1$ is used in writing $A_{Q}^{P}$. Lemma 5.6 yields that $\mathcal{B}^{\infty}$ is imbedded in $B_{Q+P(b-a)}^{p}$ by the inclusion map. By Theorem 5.10, this implies $a<b+(1+Q) / P$.

The fourth group consists of the case $\langle 6\rangle$ only. Let $H \in \mathcal{B}_{a-b}^{\infty}$; then $D_{a}^{b-a} H=h \in \mathcal{B}^{\infty}$ by Theorem 4.2. Exactly as in the proof of the third group of cases, $h \in B_{b}^{1}$. Let $t>0$, that is, satisfy (8) with $\alpha=0$. Together with the first necessary condition, this gives $b+t>-1$. Now consider the sequence of bounded maps

$$
\mathcal{B}_{a-b}^{\infty} \stackrel{D_{a}^{b-a}}{\longrightarrow} \mathcal{B}^{\infty} \stackrel{I_{b}^{t}}{\longrightarrow} L^{\infty} \stackrel{T_{a b}}{\longrightarrow} L^{\infty} \cap H(\mathbb{B})=H^{\infty} .
$$

Lemma 5.6 yields that $\mathcal{B}_{a-b}^{\infty}$ is imbedded in $H^{\infty}$ by the inclusion map. By Theorem 5.11, this implies $a<b$.

Thirdly, we prove that in the cases $\langle 2\rangle,\langle 3\rangle,\langle 4\rangle$, if one of the inequalities in (III) is an equality, then the other must be a strict inequality.

Theorem 6.3. Let $a, b, q, Q \in \mathbb{R}$ and $1 \leq p, P \leq \infty$. If $T_{a b}: L_{q}^{p} \rightarrow L_{Q}^{P}$ is bounded, then equality cannot hold simultaneously in the inequalities of Theorems 6.1 and 6.2 in the cases $\langle 2\rangle,\langle 3\rangle,\langle 4\rangle$.

Proof. The boundedness of $T_{a b}$ via Corollary 4.11 implies $Q>-1$ when $P<\infty$.

If $q=b$ and $a=b+Q-q$ simultaneously in the case $\langle 2\rangle$, then also $a=Q>-1$ and $T_{a b}^{*}=T_{Q Q}: L^{\infty} \rightarrow L^{\infty}$ is bounded. Let

$$
g_{z}(w)=\frac{(1-\langle z, w\rangle)^{1+N+Q}}{|1-\langle z, w\rangle|^{1+N+Q}}
$$

which is a uniformly bounded family for $z \in \mathbb{B}$. The same is true also of $\left\{T_{Q Q} g_{z}\right\}$. But

$$
T_{Q Q} g_{z}(z)=\int_{\mathbb{B}} \frac{\left(1-|w|^{2}\right)^{Q}}{|1-\langle z, w\rangle|^{1+N+Q}} d \nu(w) \sim \frac{1}{|z|^{2}} \log \frac{1}{1-|z|^{2}}
$$

by Proposition 5.4, contradicting uniform boundedness. 
If $q=b$ and $a=b+(1+N+Q) / P-(1+N+q)$ simultaneously in the case $\langle 3\rangle$, then also $a=(1+N+Q) / P-(1+N)$ and $T_{a b}^{*}=T_{a Q}: L_{Q}^{P^{\prime}} \rightarrow L^{\infty}$ is bounded with $P^{\prime}>1$. There is an unbounded $g \in B_{-(1+N)}^{P^{\prime}}$ by Theorem 5.9. Then $h=D_{Q-(1+N+Q) / P^{\prime}}^{(1+N+Q) / P^{\prime}} g \in B_{Q}^{P^{\prime}} \subset L_{Q}^{P^{\prime}} \subset L_{Q}^{1}$. By Lemma 5.5 and (4), we obtain

$$
T_{a b}^{*} h=T_{a Q} h=C D_{Q}^{a-Q} h=C D_{Q}^{a-Q} D_{Q-(1+N+Q) / P^{\prime}}^{(1+N+Q) / P^{\prime}} g=C g
$$

since $(a-Q)+(1+N+Q) / P^{\prime}=0$ by the conditions on the parameters. But $g \notin L^{\infty}$, and this contradicts that $T_{a b}^{*}: L_{Q}^{P^{\prime}} \rightarrow L^{\infty}$.

If $q=b$ and $a=b-(1+N+q)$ simultaneously in the case $\langle 4\rangle$, then also $a=-(1+N)$. For $j=1,2, \ldots$, let $z_{j}=(1-1 / j, 0, \ldots, 0)$ and $E_{j}$ the ball of radius $1 / 2 j$ centered at $z_{j}$, and define

$$
f_{j}(z)=\frac{\chi_{E_{j}}(z)}{\nu\left(E_{j}\right)\left(1-|z|^{2}\right)^{q}} .
$$

Clearly $f_{j} \in L_{q}^{1}$ and $\left\|f_{j}\right\|_{L_{q}^{1}}=1$ for every $j$. Then $\left\{T_{a b} f_{j}\right\}=\left\{T_{-(1+N), q} f_{j}\right\}$ is a uniformly bounded family. By the mean value property,

$$
T_{-(1+N), q} f_{j}(w)=\overline{\frac{1}{\nu\left(E_{j}\right)} \int_{E_{j}} K_{-(1+N)}(z, w) d \nu(z)}=K_{-(1+N)}\left(w, z_{j}\right) .
$$

But

$$
T_{-(1+N), q} f_{j}\left(z_{j}\right)=K_{-(1+N)}\left(z_{j}, z_{j}\right)=\frac{1}{\left|z_{j}\right|^{2}} \log \frac{1}{1-\left|z_{j}\right|^{2}}
$$

contradicting uniform boundedness.

\section{Sufficiency Proofs}

Conversely, we now present the proofs that the two inequalities in (III) of Theorems 1.2 and 1.3 imply the boundedness of $S_{a b}$. By Lemma 4.12, it suffices to prove this only for large values of $a$. In all the cases except $\langle 4\rangle$, there are values of $a>-(1+N)$ satisfying the inequalities in (III). So in this section we make the standing hypothesis

$$
a>-(1+N) \text { and } K_{a} \text { is binomial except in case }\langle 4\rangle \text {. }
$$

In the case $\langle 4\rangle$, the remaining values of $a$ are handled separately and swiftly.

Proof of Sufficiency. Each of the ten cases has a sufficiently different proof from those of the other cases and we treat each case separately. The cases $\langle 7\rangle$ and $\langle 1\rangle$ are the generic cases of Theorems 1.3 and Theorem 1.2, respectively, and have the most involved proofs, so we leave them to the end. Throughout, our hypothesis is that the two inequalities in (III) hold. 
Case $\langle\mathbf{2}\rangle$. Let $f \in L_{q}^{1}$. We write the $L_{Q}^{1}$ norm of $S_{a b} f$ explicitly, then exchange the order of integration by the Fubini theorem, and obtain

$$
\begin{aligned}
\left\|S_{a b} f\right\|_{L_{Q}^{1}} & \leq \int_{\mathbb{B}} \int_{\mathbb{B}}\left|K_{a}(w, z)\right||f(z)|\left(1-|z|^{2}\right)^{b} d \nu(z)\left(1-|w|^{2}\right)^{Q} d \nu(w) \\
& =\int_{\mathbb{B}}|f(z)|\left(1-|z|^{2}\right)^{b} \int_{\mathbb{B}} \frac{\left(1-|w|^{2}\right)^{Q}}{|1-\langle z, w\rangle|^{1+N+a}} d \nu(w) d \nu(z) \\
& =\int_{\mathbb{B}}|f(z)|\left(1-|z|^{2}\right)^{b-q} \int_{\mathbb{B}} \frac{\left(1-|w|^{2}\right)^{Q}}{|1-\langle z, w\rangle|^{1+N+a}} d \nu(w) d \nu_{q}(z)
\end{aligned}
$$

Let $J(z)$ be that part of the integrand of the outer integral multiplying $|f(z)|$. We show that $J$ is bounded on $\mathbb{B}$ using Proposition 5.4. Check that $Q>-1$ as required.

Consider $(1+N+a)-(1+N)-Q=a-Q$. If $a-Q<0$, then the integral in $J(z)$ is bounded and $J(z)$ is also bounded since $b-q \geq 0$ by the first inequality in (III). If $a-Q=0$, then the integral in $J(z)$ is logarithmic. But now $b>q$ by (III) and Remark 1.6, and $J(z)$ is bounded this time by (12). If $a-Q>0$, then $J(z) \sim\left(1-|z|^{2}\right)^{b-q-a+Q}$. But $b-q-a+Q \geq 0$ by the second inequality in (III); hence $J(z)$ is bounded once again. Thus $\left\|S_{a b} f\right\|_{L_{Q}^{1}} \lesssim\|f\|_{L_{q}^{1}}$ and $S_{a b}: L_{q}^{1} \rightarrow L_{Q}^{1}$ is bounded.

Case $\langle\mathbf{3}\rangle$. Let $f \in B_{q}^{1}$. We write the $L_{Q}^{P}$ norm of $S_{a b} f$ explicitly, then use Lemma 5.3 with the measures $\nu_{q}$ and $\nu_{Q}$, and obtain

$$
\begin{aligned}
\left\|S_{a b} f\right\|_{L_{Q}^{P}} & =\left(\int_{\mathbb{B}}\left|\int_{\mathbb{B}} K_{a}(w, z) f(z)\left(1-|z|^{2}\right)^{b-q} d \nu_{q}(z)\right|^{P} d \nu_{Q}(w)\right)^{1 / P} \\
& \leq \int_{\mathbb{B}}\left(\int_{\mathbb{B}}\left|K_{a}(w, z)\right|^{P}|f(z)|^{P}\left(1-|z|^{2}\right)^{(b-q) P} d \nu_{Q}(w)\right)^{1 / P} d \nu_{q}(z) \\
& =\int_{\mathbb{B}}|f(z)|\left(1-|z|^{2}\right)^{b-q}\left(\int_{\mathbb{B}} \frac{\left(1-|w|^{2}\right)^{Q}}{|1-\langle z, w\rangle|^{(1+N+a) P}} d \nu(w)\right)^{1 / P} d \nu_{q}(z) .
\end{aligned}
$$

For $z \in \mathbb{B}$, let $J(z)$ be that part of the integrand of the outer integral multiplying $|f(z)|$. We show that $J$ is bounded on $\mathbb{B}$ using Proposition 5.4. Check that $Q>-1$ as required.

Let $u=(1+N+a) P-(1+N)-Q$. If $u<0$, then the integral in $J(z)$ is bounded and $J(z)$ is also bounded since $b-q \geq 0$ by the first inequality in (III). If $u=0$, then the integral in $J(z)$ is logarithmic and $J(z)$ is bounded by (12) since (III) reads $q<b$ by Remark 1.6 with this value of $u$. If $u>0$, then $J(z) \sim\left(1-|z|^{2}\right)^{b-q-u / P}$. Note that

$$
b-q-\frac{u}{P}=b-q-(1+N+a)+\frac{1+N+Q}{P} \geq 0
$$


by the second inequality in (III). So $J(z)$ is bounded one more time. Thus $\left\|S_{a b} f\right\|_{L_{Q}^{P}} \lesssim\|f\|_{L_{q}^{1}}$ and $S_{a b}: L_{q}^{1} \rightarrow L_{Q}^{P}$ is bounded.

Case $\langle 4\rangle$. Let $f \in L_{q}^{1}$. If $q=b$, then (III) says that $a<-(1+N)$ and $K_{a}$ is bounded by Lemma 4.1 .

$$
\left|S_{a b} f(w)\right| \leq \int_{\mathbb{B}}\left|K_{a}(w, z)\right||f(z)|\left(1-|z|^{2}\right)^{b} d \nu(z) \lesssim\|f\|_{L_{q}^{1}} \quad(w \in \mathbb{B}) .
$$

Then $\left\|S_{a b} f\right\|_{L^{\infty}} \lesssim\|f\|_{L_{q}^{1}}$.

Otherwise $q<b$ and there are values of $a>-(1+N)$ satisfying the inequalities in (III). So in the rest of this case we can assume $a>-(1+N)$ and $K_{a}$ is binomial by Lemma 4.12. Then writing $S_{a b} f(w)$ explicitly and simple manipulations give

$$
\begin{aligned}
\left|S_{a b} f(w)\right| & \leq \int_{\mathbb{B}}\left|K_{a}(w, z)\right||f(z)|\left(1-|z|^{2}\right)^{b} d \nu(z) \\
& =\int_{\mathbb{B}}|f(z)| \frac{\left(1-|z|^{2}\right)^{b-q}}{|1-\langle w, z\rangle|^{1+N+a}} d \nu_{q}(z)=: \int_{\mathbb{B}}|f(z)| J(z, w) d \nu_{q}(z) .
\end{aligned}
$$

Since $|\langle w, z\rangle| \leq|w||z| \leq|z|$ for $w \in \mathbb{B}$, we have $J(z, w) \lesssim\left(1-|z|^{2}\right)^{b-q-(1+N+a)}$ for all such $w$. Note that the power here is nonnegative by the second inequality in (III) yielding that $J(z, w)$ is bounded for all $z, w \in \mathbb{B}$. Then we obtain

$$
\left|S_{a b} f(w)\right| \lesssim \int_{\mathbb{B}}|f(z)| d \nu_{q}(z)=\|f\|_{L_{q}^{1}} \quad(w \in \mathbb{B})
$$

and $\left\|S_{a b} f\right\|_{L^{\infty}} \lesssim\|f\|_{L_{q}^{1}}$. Thus $S_{a b}: L_{q}^{1} \rightarrow L^{\infty}$ is bounded.

Case $\langle\mathbf{5}\rangle$. Let $f \in L_{q}^{p}$. We write $S_{a b} f(w)$ explicitly, apply the Hölder inequality with the measure $\nu_{q}$, and obtain

$$
\begin{aligned}
\left|S_{a b} f(w)\right| & \leq \int_{\mathbb{B}}\left|K_{a}(w, z)\right||f(z)|\left(1-|z|^{2}\right)^{b} d \nu(z) \\
& =\int_{\mathbb{B}}|f(z)| \frac{\left(1-|z|^{2}\right)^{b-q}}{|1-\langle w, z\rangle|^{1+N+a}} d \nu_{q}(z) \\
& \leq\|f\|_{L_{q}^{p}}\left(\int_{\mathbb{B}} \frac{\left(1-|z|^{2}\right)^{(b-q) p^{\prime}+q}}{|1-\langle w, z\rangle|^{(1+N+a) p^{\prime}}} d \nu(z)\right)^{1 / p^{\prime}}=: J(w)^{1 / p^{\prime}}\|f\|_{L_{q}^{p}}
\end{aligned}
$$

We show that $J$ is bounded on $\mathbb{B}$ by Proposition 5.4. Now

$$
(b-q) p^{\prime}+q+1=p^{\prime}\left(b-q+\frac{(q+1)(p-1)}{p}\right)=p^{\prime}\left(1+b-\frac{1+q}{p}\right)>0
$$

by the first inequality in (III), so the power on $1-|z|^{2}$ in $J(w)$ is $>-1$ as required.

Note that

$$
\begin{aligned}
(1+N+a) p^{\prime}-(1+N)-(b-q) p^{\prime}-q & =p^{\prime}\left(1+N+a-b+q-\frac{1+N+q}{p^{\prime}}\right) \\
& =p^{\prime}\left(a-b+\frac{1+N+q}{p}\right)<0
\end{aligned}
$$


by the second inequality in (III). This shows that $J(w)$ is bounded for all $w \in \mathbb{B}$. Then $\left|S_{a b} f(w)\right| \lesssim\|f\|_{L_{q}^{p}}$ for all $w \in \mathbb{B}$ and $\left\|S_{a b} f\right\|_{L^{\infty}} \lesssim\|f\|_{L_{q}^{p}}$. Thus $S_{a b}: L_{q}^{p} \rightarrow L^{\infty}$ is bounded.

Case $\langle\mathbf{6}\rangle$. Let $f \in L^{\infty}$. We write $S_{a b} f(w)$ explicitly, take the $L^{\infty}$ norm of $f$ out of the integral, and obtain

$$
\begin{aligned}
\left|S_{a b} f(w)\right| & \leq \int_{\mathbb{B}}\left|K_{a}(w, z)\right||f(z)|\left(1-|z|^{2}\right)^{b} d \nu(z) \\
& \leq\|f\|_{L^{\infty}} \int_{\mathbb{B}} \frac{\left(1-|z|^{2}\right)^{b}}{|1-\langle w, z\rangle|^{1+N+a}} d \nu(z)=: J(w)\|f\|_{L^{\infty}} .
\end{aligned}
$$

We show that $J$ is bounded on $\mathbb{B}$ by Proposition 5.4. Check that $b>-1$ by the first inequality in (III). The second inequality in (III) gives that $a<b$ and hence $J(w)$ is indeed bounded for $w \in \mathbb{B}$. Then we obtain $\left|S_{a b} f(w)\right| \lesssim\|f\|_{L^{\infty}}$ for all $w \in \mathbb{B}$. Thus $\left\|S_{a b} f\right\|_{L^{\infty}} \lesssim\|f\|_{L_{q}^{p}}$ and $S_{a b}: L^{\infty} \rightarrow L^{\infty}$ is bounded.

Case $\langle 8\rangle$. Let $f \in L_{q}^{p}$. We write the $L_{Q}^{1}$ norm of $S_{a b} f$ explicitly, then exchange the order of integration by the Fubini theorem, afterwards apply the Hölder inequality, and obtain

$$
\begin{aligned}
\left\|S_{a b} f\right\|_{L_{Q}^{1}} & \leq \int_{\mathbb{B}} \int_{\mathbb{B}}\left|K_{a}(w, z)\right||f(z)|\left(1-|z|^{2}\right)^{b} d \nu(z)\left(1-|w|^{2}\right)^{Q} d \nu(w) \\
& =\int_{\mathbb{B}}|f(z)|\left(1-|z|^{2}\right)^{b} \int_{\mathbb{B}} \frac{\left(1-|w|^{2}\right)^{Q}}{|1-\langle z, w\rangle|^{1+N+a}} d \nu(w) d \nu(z) \\
& =\int_{\mathbb{B}}|f(z)|\left(1-|z|^{2}\right)^{q / p} \int_{\mathbb{B}} \frac{\left(1-|w|^{2}\right)^{Q} d \nu(w)}{|1-\langle z, w\rangle|^{1+N+a}}\left(1-|z|^{2}\right)^{b-q / p} d \nu(z) \\
& \leq\|f\|_{L_{q}^{p}}\left(\int_{\mathbb{B}}\left(\int_{\mathbb{B}} \frac{\left(1-|w|^{2}\right)^{Q} d \nu(w)}{|1-\langle z, w\rangle|^{1+N+a}}\right)^{p^{\prime}}\left(1-|z|^{2}\right)^{(b-q / p) p^{\prime}} d \nu(z)\right)^{1 / p^{\prime}} \\
& =: J^{1 / p^{\prime}}\|f\|_{L_{q}^{p}}
\end{aligned}
$$

We show that $J$ is finite using Proposition 5.4. Check that $Q>-1$ as required.

If $a<Q$, then $J \sim \int_{\mathbb{B}}\left(1-|z|^{2}\right)^{(b-q / p) p^{\prime}} d \nu(z)$. Note that

$$
\left(b-\frac{q}{p}\right) p^{\prime}=p^{\prime}\left(1+b-\frac{1+q}{p}-\frac{1}{p^{\prime}}\right)>p^{\prime}\left(-\frac{1}{p^{\prime}}\right)=-1
$$

by the first inequality in (III). So $J$ is finite by Lemma 4.6. If $a=Q$, then

$$
J \sim \int_{\mathbb{B}}\left(\frac{1}{|z|^{2}} \log \frac{1}{1-|z|^{2}}\right)^{p^{\prime}}\left(1-|z|^{2}\right)^{(b-q / p) p^{\prime}} d \nu(z)<\infty
$$

also by Lemma 4.6. If $a>Q$, then $J \sim \int_{\mathbb{B}}\left(1-|z|^{2}\right)^{(b-q / p) p^{\prime}-(a-Q) p^{\prime}} d \nu(z)$. But

$$
\left(b-\frac{q}{p}\right) p^{\prime}-(a-Q) p^{\prime}=p^{\prime}\left(b-a+1+Q-\frac{1+q}{p}-\frac{1}{p^{\prime}}\right)>p^{\prime}\left(-\frac{1}{p^{\prime}}\right)=-1
$$


by the second inequality in (III). So $J$ is finite one more time. We conclude that $S_{a b}: L_{q}^{p} \rightarrow L_{Q}^{1}$ is bounded.

Case $\langle\mathbf{9}\rangle$. Let $f \in L^{\infty}$. We write the $L_{Q}^{1}$ norm of $S_{a b} f$ explicitly, then exchange the order of integration by the Fubini theorem, take the $L^{\infty}$ norm of $f$ out of the integral, and obtain

$$
\begin{aligned}
\left\|S_{a b} f\right\|_{L_{Q}^{1}} & \leq \int_{\mathbb{B}} \int_{\mathbb{B}}\left|K_{a}(w, z)\right||f(z)|\left(1-|z|^{2}\right)^{b} d \nu(z)\left(1-|w|^{2}\right)^{Q} d \nu(w) \\
& =\int_{\mathbb{B}}|f(z)|\left(1-|z|^{2}\right)^{b} \int_{\mathbb{B}} \frac{\left(1-|w|^{2}\right)^{Q}}{|1-\langle z, w\rangle|^{1+N+a}} d \nu(w) d \nu(z) \\
& \leq\|f\|_{L^{\infty}} \int_{\mathbb{B}}\left(1-|z|^{2}\right)^{b} \int_{\mathbb{B}} \frac{\left(1-|w|^{2}\right)^{Q}}{|1-\langle z, w\rangle|^{1+N+a}} d \nu(w) d \nu(z) \\
& =: J\|f\|_{L^{\infty}} .
\end{aligned}
$$

We show that $J$ is finite using Proposition 5.4. Check that $Q>-1$ as required.

If $a<Q$, then the inner integral in $J$ is bounded and hence $J$ is finite by the first inequality in (III). If $a=Q$, then the inner integral is logarithmic and hence $J$ is finite by the first inequality in (III) and Lemma 4.6. If $a>Q$, then $J \sim \int_{\mathbb{B}}\left(1-|z|^{2}\right)^{b-a+Q} d \nu(z)<\infty$ since $b-a+Q>-1$ by the second inequality in (III). Thus $S_{a b}: L^{\infty} \rightarrow L_{Q}^{1}$ is bounded.

Case $\langle\mathbf{1 0}\rangle$. Let $f \in L^{\infty}$. We write the $L_{Q}^{P}$ norm of $S_{a b} f$ explicitly, take the $L^{\infty}$ norm of $f$ out of the integral, and obtain

$$
\begin{aligned}
\left\|S_{a b} f\right\|_{L_{Q}^{P}}^{P} & =\int_{\mathbb{B}}\left|\int_{\mathbb{B}} K_{a}(w, z) f(z)\left(1-|z|^{2}\right)^{b} d \nu(z)\right|^{P}\left(1-|w|^{2}\right)^{Q} d \nu(w) \\
& \leq\|f\|_{L^{\infty}}^{P} \int_{\mathbb{B}}\left(1-|w|^{2}\right)^{Q}\left(\int_{\mathbb{B}} \frac{\left(1-|z|^{2}\right)^{b}}{|1-\langle w, z\rangle|^{1+N+a}} d \nu(z)\right)^{P} d \nu(w) \\
& =: J\|f\|_{L^{\infty}}^{P} .
\end{aligned}
$$

We show that $J$ is finite using Proposition 5.4. Check that $b>-1$ by the first inequality in (III) as required.

If $a<b$, then the inner integral in $J$ is bounded and then $J$ is finite since $Q>-1$. If $a=b$, then the inner integral in $J$ is logarithmic and then $J$ is finite by Lemma 4.6. If $a>b$, then $J \sim \int_{\mathbb{B}}\left(1-|w|^{2}\right)^{Q-(a-b) P} d \nu(w)$. Note that

$$
Q-(a-b) P=Q-(a-b) P+1-1=P\left(\frac{1+Q}{P}+b-a\right)-1>-1
$$

by the second inequality in (III), which shows that $J$ is again finite. Consequently $S_{a b}: L^{\infty} \rightarrow L_{Q}^{P}$ is bounded.

Case $\langle 7\rangle$. We employ the Schur test in Theorem 5.2 with the measures $\lambda=\nu_{q}, \mu=\nu_{Q}$ and the kernel $G(z, w)=\frac{\left(1-|z|^{2}\right)^{b-q}}{|1-\langle z, w\rangle|^{1+N+a}}$ which together give us $Z=S_{a b}$, along with the strictly positive functions $\phi(z)=\left(1-|z|^{2}\right)^{m}$ 
and $\psi(w)=\left(1-|w|^{2}\right)^{n}$ on $\mathbb{B}$ with $m, n \in \mathbb{R}$ to be determined. Two of the three conditions that need to be satisfied for the test are

$$
\begin{gathered}
\int_{\mathbb{B}} \frac{\left(1-|z|^{2}\right)^{b-q}}{|1-\langle w, z\rangle|^{1+N+a}}\left(1-|z|^{2}\right)^{m p^{\prime}}\left(1-|z|^{2}\right)^{q} d \nu(z) \lesssim\left(1-|w|^{2}\right)^{n P^{\prime}}, \\
\int_{\mathbb{B}} \frac{\left(1-|z|^{2}\right)^{b-q}}{|1-\langle w, z\rangle|^{1+N+a}}\left(1-|w|^{2}\right)^{n P}\left(1-|w|^{2}\right)^{Q} d \nu(w) \lesssim\left(1-|z|^{2}\right)^{m p}
\end{gathered}
$$

One way to satisfy them is by matching the growth rates of their two sides, that is, the powers of the $1-|\cdot|^{2}$. By Proposition 5.4, this is possible if $m, n<0$ and

$$
\begin{aligned}
-n P^{\prime} & =a-\left(b+m p^{\prime}\right), \\
-m p & =a-(n P+Q)-(b-q) .
\end{aligned}
$$

But we must also make sure that the conditions of Proposition 5.4 for this to happen are met, that is,

$$
\begin{array}{rlr}
b+m p^{\prime} & >-1, \quad n P+Q & >-1, \\
a-\left(b+m p^{\prime}\right) & >0, \quad a-(n P+Q) & >0 .
\end{array}
$$

Substituting for $p^{\prime}, P^{\prime}$ in terms of $p, P$, we can write (15) as a system of two linear equations in the two unknowns $m, n$ as

$$
\begin{aligned}
p(P-1) m-P(p-1) n & =(a-b)(p-1)(P-1), \\
-p m+P n & =a-b+q-Q .
\end{aligned}
$$

This system has the unique solution

$$
\begin{aligned}
& m=\frac{(p-1)(P(a-b)+q-Q)}{p(P-p)}, \\
& n=\frac{(P-1)(p(a-b)+q-Q)}{P(P-p)}
\end{aligned}
$$

for $m, n$. The second inequality in (III) can be written in the form

$$
a-b=-\varepsilon+\frac{1+Q}{P}-\frac{1+q}{p}
$$

with $\varepsilon>0$. By Lemma 4.12, it suffices to show that $S_{a b}$ is bounded when (19) holds for small enough $\varepsilon>0$. Substituting this value of $a-b$ into (18), the solution takes the form

$$
\begin{aligned}
& m=\frac{p-1}{p}\left(-\frac{1+q}{p}+\frac{\varepsilon P}{p-P}\right), \\
& n=\frac{P-1}{P}\left(-\frac{1+Q}{P}+\frac{\varepsilon p}{p-P}\right),
\end{aligned}
$$

What is left is to show that this solution satisfies all the required conditions for sufficiently small $\varepsilon>0$. Bear in mind that $Q>-1$. First, by the first inequality in (III),

$$
a=-\varepsilon+b+\frac{1+Q}{P}-\frac{1+q}{p}>-\varepsilon+\frac{1+Q}{P}-1>-(1+\varepsilon)>-(1+N)
$$


provided $\varepsilon<N$. Next we verify the four inequalities in (16). By (20) and the first inequality in (III),

$$
b+m p^{\prime}=b-\frac{1+q}{p}+\frac{\varepsilon P}{p-P}>-1+\frac{\varepsilon P}{p-P}>-1 .
$$

By $(20)$,

$$
n P+Q=(P-1)\left(-\frac{1+Q}{P}+\frac{\varepsilon p}{p-P}\right)+Q=-1+\frac{1+Q}{P}+\frac{\varepsilon p(P-1)}{p-P}>-1 \text {. }
$$

By (19) and (21),

$$
a-\left(b+m p^{\prime}\right)=-\varepsilon+\frac{1+Q}{P}-\frac{\varepsilon P}{p-P}=\frac{1+Q}{P}-\frac{\varepsilon p}{p-P}>0
$$

provided $\varepsilon<\left(\frac{1}{P}-\frac{1}{p}\right)(1+Q)$. By (19), (22), and the first inequality in (III),

$$
\begin{aligned}
a-(n P+Q) & =-\varepsilon+b+\frac{1+Q}{P}-\frac{1+q}{p}+1-\frac{1+Q}{P}-\frac{\varepsilon p(P-1)}{p-P} \\
& =1+b-\frac{1+q}{p}-\frac{\varepsilon P(p-1)}{p-P}>0
\end{aligned}
$$

provided $\varepsilon<\frac{p}{p-1}\left(\frac{1}{P}-\frac{1}{p}\right)\left(1+b-\frac{1+q}{p}\right)$. Lastly, we check the third condition of Theorem 5.2, which is the finiteness of

$\int_{\mathbb{B}} \int_{\mathbb{B}} \frac{\left(1-|z|^{2}\right)^{b-q}}{|1-\langle w, z\rangle|^{1+N+a}}\left(1-|z|^{2}\right)^{m p^{\prime}}\left(1-|w|^{2}\right)^{n P}\left(1-|z|^{2}\right)^{q}\left(1-|w|^{2}\right)^{Q} d \nu(z) d \nu(w)$.

Call the double integral $J$. We estimate first the integral, say, with respect to $d \nu(z)$ by Proposition 5.4 and obtain

$$
J \sim \int_{\mathbb{B}}\left(1-|w|^{2}\right)^{n P+Q-a+b+m p^{\prime}} d \nu(w)
$$

by (23). But by (22) and (23), the power on $1-|w|^{2}$ is

$$
\begin{aligned}
n P+Q-\left(a-\left(b+m p^{\prime}\right)\right) & =-1+\frac{1+Q}{P}+\frac{\varepsilon p(P-1)}{p-P}-\frac{1+Q}{P}+\frac{\varepsilon p}{p-P} \\
& =-1+\frac{\varepsilon p P}{p-P}>-1,
\end{aligned}
$$

making $J<\infty$. Thus for

$$
0<\varepsilon<\min \left\{N,\left(\frac{1}{P}-\frac{1}{p}\right)(1+Q), \frac{p}{p-1}\left(\frac{1}{P}-\frac{1}{p}\right)\left(1+b-\frac{1+q}{p}\right)\right\},
$$

Theorem 5.2 using the selected $\phi$ and $\psi$ with the powers in (20) applies and proves that $S_{a b}: L_{q}^{p} \rightarrow L_{Q}^{P}$ with $1<P<p<\infty$ is bounded when the two inequalities in (III) hold.

Case $\langle\mathbf{1}\rangle$. The proof of this case follows the same lines as the proof of $[18$, Lemma 6] and also starts out as in the proof of the case $\langle 7\rangle$. We assume (III) and take $a$ to have its largest value

$$
a=b+\frac{1+N+Q}{P}-\frac{1+N+q}{p}
$$


by Lemma 4.12 with no loss of generality. We have $a>-(1+N)$ by the first inequality in (III) and $Q>-1$. Now we employ the Schur test in Theorem 5.1 but with the same test data as in the case $\langle 7\rangle$. So we have the measures $\lambda=\nu_{q}, \mu=\nu_{Q}$ and the kernel $G(z, w)=\frac{\left(1-|z|^{2}\right)^{b-q}}{|1-\langle z, w\rangle|^{1+N+a}}$ which together give $Z=S_{a b}$, along with the strictly positive functions $\phi(z)=\left(1-|z|^{2}\right)^{m}$ and $\psi(w)=\left(1-|w|^{2}\right)^{n}$ on $\mathbb{B}$ with $m, n \in \mathbb{R}$ to be determined. The two conditions that need to be satisfied for the test are

$$
\begin{gathered}
\int_{\mathbb{B}} \frac{\left(1-|z|^{2}\right)^{(b-q) c p^{\prime}}}{|1-\langle w, z\rangle|^{(1+N+a) c p^{\prime}}}\left(1-|z|^{2}\right)^{m p^{\prime}}\left(1-|z|^{2}\right)^{q} d \nu(z) \lesssim\left(1-|w|^{2}\right)^{n p^{\prime}}, \\
\int_{\mathbb{B}} \frac{\left(1-|z|^{2}\right)^{(b-q) d P}}{|1-\langle w, z\rangle|^{(1+N+a) d P}}\left(1-|w|^{2}\right)^{n P}\left(1-|w|^{2}\right)^{Q} d \nu(w) \lesssim\left(1-|z|^{2}\right)^{m P} .
\end{gathered}
$$

One way to satisfy them is by matching the growth rates of their two sides, that is, the powers of the $1-|\cdot|^{2}$. By Proposition 5.4, this is possible if $m, n<0$ and

$$
\begin{aligned}
-n p^{\prime} & =(1+N+a) c p^{\prime}-(1+N)-(b-q) c p^{\prime}-m p^{\prime}-q, \\
-m P & =(1+N+a) d P-(1+N)-(n P+Q)-(b-q) d P .
\end{aligned}
$$

But we must also make sure that the conditions of Proposition 5.4 for this to happen are met, that is,

$$
\begin{aligned}
E:=(b-q) c p^{\prime}+m p^{\prime}+q & >-1, \\
n P+Q & >-1, \\
F:=(1+N+a) c p^{\prime}-(1+N)-(b-q) c p^{\prime}-m p^{\prime}-q & >0, \\
(1+N+a) d P-(1+N)-(n P+Q) & >0 .
\end{aligned}
$$

If we were to continue as in the proof of the case $\langle 7\rangle$, we would now solve for $m, n$ from the linear equations in (25). However, it turns out that these two equations are linearly dependent, so we follow a different path. We first pick an $n$ to satisfy the second inequality in (26); so

$$
-\frac{1+Q}{P}<n<0
$$

which is possible since $Q>-1$. Next we pick a $d$ to satisfy the fourth inequality in (26) and naturally let $c=1-d$; so we take

$$
\begin{aligned}
& d=\frac{1}{1+N+a}\left(n+\frac{1+N+Q}{P}+\varepsilon\right) \\
& c=\frac{1}{1+N+a}\left(-n+1+N+b-\frac{1+N+q}{p}-\varepsilon\right)
\end{aligned}
$$

with $\varepsilon>0$ by (24). Using the chosen values of $n, c, d$, we then solve for $m$ from, say, the second equation in (25), and simplify it using the definition of $d ;$ so

$$
\begin{aligned}
m & =(b-q) d-(1+N+a) d+n+\frac{1+N+Q}{P} \\
& =(b-q) d-n-\frac{1+N+Q}{P}-\varepsilon+n+\frac{1+N+Q}{P}=(b-q) d-\varepsilon .
\end{aligned}
$$


Finally, we make sure the remaining first and third inequalities in (26) hold for some $\varepsilon>0$. Substituting in the value of $m$ from $(29)$, since $c+d=1$,

$$
\begin{aligned}
E+1 & =(b-q) c p^{\prime}+(b-q) d p^{\prime}-\varepsilon p^{\prime}+q+1=(b-q) p^{\prime}+q+1-\varepsilon p^{\prime} \\
& =p^{\prime}\left(b-q+\frac{q}{p^{\prime}}+\frac{1}{p^{\prime}}\right)-\varepsilon p^{\prime}=p^{\prime}\left(b-\frac{q}{p}+1-\frac{1}{p}\right)-\varepsilon p^{\prime} \\
& =p^{\prime}\left(1+b-\frac{1+q}{p}-\varepsilon\right)>0
\end{aligned}
$$

by the first inequality in (III) provided $\varepsilon<1+b-\frac{1+q}{p}$. Again since $c+d=1$ and substituting in for $m$ and $c$ from (29) and (28),

$$
\begin{aligned}
F & =p^{\prime}\left((1+N+a) c-(b-q) c-(b-q) d+\varepsilon-\frac{1+N+q}{p^{\prime}}\right) \\
& =p^{\prime}\left(-n+1+N+b-\frac{1+N+q}{p}-\varepsilon-b+q+\varepsilon-\frac{1+N+q}{p^{\prime}}\right) \\
& =-p^{\prime} n>0
\end{aligned}
$$

by (27). Thus for

$$
0<\varepsilon<1+b-\frac{1+q}{p},
$$

Theorem 5.1 using the selected $\phi$ and $\psi$ with the powers in (29) and (27) applies with the parameters in (28) and proves that $S_{a b}: L_{q}^{p} \rightarrow L_{Q}^{P}$ with $1<p \leq P<\infty$ is bounded when the two inequalities in (III) hold.

As added information, from (28) and (27), we see that

$$
d>\frac{1}{1+N+a}\left(-\frac{1+Q}{P}+\frac{1+N+Q}{P}+\varepsilon\right)=\frac{1}{1+N+a}\left(\frac{N}{P}+\varepsilon\right)>0,
$$

but we do not see any such relation for $c=1-d$.

\section{Mapping into Bergman-Besov Spaces and $H^{\infty}$}

We now prove Theorem 1.8. Having proved Theorems 1.2 and 1.3, our task is easy with the technique of composing maps employed in Theorem 6.2. The replacement we need for Lemma 5.6 is Lemma 5.7.

Proof of Theorem 1.8. First Necessary Condition. We imitate the proof of Theorem 6.1 assuming $T_{a b}$ is bounded. We form the same three groups of the ten cases and use the same test functions $f_{u v}$ for each group obtaining $T_{a b} f_{u v} \in B_{Q}^{P}$. Then $T_{a b} f_{u v}(0) \in \mathbb{C}$. In the first group excluding the case $\langle 5\rangle$,

$$
T_{a b} f_{u v}(0)=\int_{\mathbb{B}}\left(1-|z|^{2}\right)^{b-(1+q) / p}\left(\frac{1}{|z|^{2}} \log \frac{1}{1-|z|^{2}}\right)^{-1} d \nu(z) .
$$

Writing the integral in polar coordinates and using Lemma 4.6, we obtain $(1+q) / p<1+b$. In the second group excluding the case $\langle 4\rangle$,

$$
T_{a b} f_{u 0}(0)=\int_{\mathbb{B}}\left(1-|z|^{2}\right)^{b+u} d \nu(z)
$$


with $u>-(1+q)$. Writing this in polar coordinates and using Lemma 4.6, we obtain $q \leq b$. In the third group excluding the case $\langle 6\rangle$,

$$
T_{a b} f_{00}(0)=\int_{\mathbb{B}}\left(1-|z|^{2}\right)^{b} d \nu(z) .
$$

Writing this in polar coordinates and using Lemma 4.6, we obtain $0<1+b$.

Second Necessary Condition. Assuming $T_{a b}$ is bounded and the first necessary condition, we use Theorems 1.2 and 1.3. Let $f \in L_{q}^{p}$. We first show also $f \in L_{b}^{1}$. In the first group, by the Hölder inequality,

$$
\begin{aligned}
\|f\|_{L_{b}^{1}} & =\int_{\mathbb{B}}|f(z)|\left(1-|z|^{2}\right)^{b-q} d \nu_{q}(z) \\
& \leq\|f\|_{L_{q}^{p}}\left(\int_{\mathbb{B}}\left(1-|z|^{2}\right)^{(b-q) p^{\prime}} d \nu_{q}(z)\right)^{1 / p^{\prime}}<\infty,
\end{aligned}
$$

because $(b-q) p^{\prime}+q=\frac{p(1+b)-(q+p)}{p-1}>\frac{1+q-(q+p)}{p-1}=-1$ by the already obtained first necessary condition. In the second group, $q \leq b$ by the first necessary condition and thus $f \in L_{b}^{1}$ by (12). In the third group, $f \in L^{\infty}$ and $b>-1$ by the first necessary condition; hence $f \in L_{b}^{1}$ again.

Now consider the composition of bounded maps

$$
L_{q}^{p} \stackrel{T_{a b}}{\longrightarrow} B_{Q}^{P} \stackrel{D_{a}^{-Q / P}}{\longrightarrow} A^{P},
$$

where Theorem 4.2 is put to work. Since we have shown $L_{q}^{p} \subset L_{b}^{1}$ in the previous paragraph, by Lemma 5.7 , this composition equals $T_{a-Q / P, b}$. We conclude that $T_{a-Q / P, b}: L_{q}^{p} \rightarrow L^{P}$ is bounded by Corollary 4.11 . Hence the second inequalities in (III) obtain with $a-Q / P$ in place of $a$ and 0 in place of $Q$. But these are exactly the same second inequalities in (III).

Sufficiency. We assume that the two inequalities in (III) hold excluding the cases $\langle 4\rangle,\langle 5\rangle,\langle 6\rangle$. As in the previous paragraph, the second inequality is equivalent to that with $a$ replaced by $a-Q / P$ and $Q$ by 0 . Now Theorems 1.2 and 1.3 and Corollary 4.11 imply that $T_{a-Q / P, b}: L_{q}^{p} \rightarrow L^{P} \cap H(\mathbb{B})=A^{P}$ is bounded. Then the composition of maps

$$
L_{q}^{p} \stackrel{T_{a-Q / P, b}}{\longrightarrow} A^{P} \stackrel{D_{a-Q / P}^{Q / P}}{\longrightarrow} B_{Q}^{P}
$$

is also bounded by Theorem 4.2. The first inequality in (III) as in the proof of the necessity part yields $L_{q}^{p} \subset L_{b}^{1}$. Then by Lemma 5.7 , the composition equals $T_{a b}: L_{q}^{p} \rightarrow B_{Q}^{P}$.

Proof of Theorem 1.9. If $T_{a b}: L_{q}^{p} \rightarrow H^{\infty}$ is bounded, then since $H^{\infty} \subset L^{\infty}$ with the same norms, $T_{a b}: L_{q}^{p} \rightarrow L^{\infty}$ is also bounded. Then Theorem 1.2 implies the first and second necessary conditions in the cases $\langle 4\rangle,\langle 5\rangle,\langle 6\rangle$.

Conversely, if the two inequalities in (III) hold in the cases $\langle 4\rangle,\langle 5\rangle,\langle 6\rangle$, then $T_{a b}: L_{q}^{p} \rightarrow L^{\infty}$ is bounded by Theorem 1.2. But Corollary 4.11 shows that the range of $T_{a b}$ actually lies in $H^{\infty}$ rendering $T_{a b}: L_{q}^{p} \rightarrow H^{\infty}$ bounded.

Remark 8.1. As a final note, let's show how our results on weighted spaces can be obtained from those on unweighted spaces. We assume now that Theorems 1.2 and 1.3 are proved in the case $q=Q=0$ and we obtain them in 
the general case with nonzero $q, Q$. We show the details only in the case $\langle 1\rangle$. We use a few results from Sects. 4 and 5 whose proofs are independent of the proofs of our main results. Let $M_{u}$ denote the operator of multiplication by $\left(1-|z|^{2}\right)^{u}$ as before. Clearly $M_{-q / p}: L^{p} \rightarrow L_{q}^{p}$ is an isometric isomorphism. Consider the sequence of bounded maps

$$
L^{p} \stackrel{M_{-q / p}}{\longrightarrow} L_{q}^{p} \stackrel{T_{a b}}{\longrightarrow} L_{Q}^{P} \cap H(\mathbb{B})=A_{Q}^{P} \stackrel{D_{a}^{-Q / P}}{\longrightarrow} A^{P},
$$

where we have $A_{Q}^{P}$ by Corollary 4.11. The last map is also an isomorphism by Theorem 4.2. The composition of the three maps is $T_{a-Q / P, b-q / p}: L^{p} \rightarrow L^{P}$ by Lemma 5.7, and it is bounded if and only if the middle map $T_{a b}: L_{q}^{p} \rightarrow L_{Q}^{P}$ is bounded. By assumption, the composition is bounded if and only if

$$
\frac{1}{p}<1+b-\frac{q}{p} \quad \text { and } \quad a-\frac{Q}{P} \leq b-\frac{q}{p}+\frac{1+N}{P}-\frac{1+N}{p},
$$

which are nothing but the inequalities in (III) of the case $\langle 1\rangle$.

Publisher's Note Springer Nature remains neutral with regard to jurisdictional claims in published maps and institutional affiliations.

\section{References}

[1] Beatrous, F., Burbea, J.: Holomorphic Sobolev spaces on the ball. Diss. Math. 276, 57 (1989)

[2] Cheng, G., Hou, X., Liu, C.: The singular integral operator induced by DruryArveson kernel. Complex Anal. Oper. Theory 12, 917-929 (2018)

[3] Cheng, G., Fang, X., Wang, Z., Yu, J.: The hyper-singular cousin of the Bergman projection. Trans. Am. Math. Soc. 369, 8643-8662 (2017)

[4] Čučković, Ž., McNeal, J.D.: Special Toeplitz operators on strongly pseudoconvex domains. Rev. Mat. Iberoam. 22, 851-866 (2006)

[5] Doğan, Ö.F., Üreyen, A.E.: Weighted harmonic Bloch spaces on the ball. Complex Anal. Oper. Theory 12, 1143-1177 (2018)

[6] Faraut, J., Korányi, A.: Function spaces and reproducing kernels on bounded symmetric domains. J. Funct. Anal. 88, 64-89 (1990)

[7] Forelli, F., Rudin, W.: Projections on spaces of holomorphic functions in balls. Indiana Univ. Math. J. 24, 593-602 (1974)

[8] Gagliardo, E.: On integral transformations with positive kernel. Proc. Am. Math. Soc. 16, 429-434 (1965)

[9] Hartz, M.: On the isomorphism problem for multiplier algebras of NevanlinnaPick spaces. Canad. J. Math. 69, 54-106 (2017)

[10] Kaptanoğlu, H.T.: Bergman projections on Besov spaces on balls. Ill. J. Math. 49, 385-403 (2005)

[11] Kaptanoğlu, H.T.: Carleson measures for Besov spaces on the ball with applications. J. Funct. Anal. 250, 483-520 (2007)

[12] Kaptanoğlu, H.T., Üreyen, A.E.: Precise inclusion relations among BergmanBesov and Bloch-Lipschitz spaces and $H^{\infty}$ on the unit ball of $\mathbb{C}^{N}$. Math. Nachr. 291, 2236-2251 (2018) 
[13] Okikiolu, G.O.: On inequalities for integral operators. Glasg. Math. J. 11, 126133 (1970)

[14] Okikiolu, G.O.: Aspects of the Theory of Bounded Integral Operators in $L^{p}$ Spaces. Academic, London (1971)

[15] Olver, F.W.J., Lozier, D.W., Boisvert, R.F., Clark, C.W. (eds.): NIST Handbook of Mathematical Functions. Cambridge Univ, New York (2010)

[16] Rudin, W.: Function Theory in the Unit Ball of $\mathbb{C}^{n}$, Grundlehren Math. Wiss., vol. 241, Springer, New York (1980)

[17] Zaharjuta, V.P., Judovič, V.I.: The general form of a linear functional in $H_{p}^{\prime}$. Uspekhi Mat. Nauk 19, 139-142 (1964)

[18] Zhao, R.: Generalization of Schur's test and its application to a class of integral operators on the unit ball of $\mathbb{C}^{n}$. Integral Equ. Oper. Theory 82, 519-532 (2015)

H. Turgay Kaptanoğlu $(\bowtie)$

Bilkent Üniversitesi, Matematik Bölümü

06800 Ankara

Turkey

e-mail: kaptan@fen.bilkent.edu.tr

URL: http://www.fen.bilkent.edu.tr/ kaptan/

A. Ersin Üreyen

Eskişehir Teknik Üniversitesi, Fen Fakültesi

Matematik Bölümü

26470 Eskisehir

Turkey

e-mail: aeureyen@eskisehir.edu.tr

URL: https://akademik.eskisehir.edu.tr/aeureyen/

Received: November 21, 2018.

Revised: May 15, 2019. 